BULLETIN (New Series) OF THE

AMERICAN MATHEMATICAL SOCIETY

Volume 50, Number 3, July 2013, Pages 391-429

S 0273-0979(2013)01410-1

Article electronically published on April 2, 2013

\title{
SINGULAR PERTURBATIONS OF COMPLEX POLYNOMIALS
}

\author{
ROBERT L. DEVANEY
}

\begin{abstract}
In this paper we describe the dynamics of singularly perturbed complex polynomials. That is, we start with a complex polynomial whose dynamics are well understood. Then we perturb this map by adding a pole, i.e., by adding in a term of the form $\lambda /(z-a)^{d}$ where the parameter $\lambda$ is complex. This changes the polynomial into a rational map of higher degree and, as we shall see, the dynamical behavior explodes.

One aim of this paper is to give a survey of the many different topological structures that arise in the dynamical and parameter planes for these singularly perturbed maps. We shall show how Sierpiński curves arise in a myriad of different ways as the Julia sets for these singularly perturbed maps, and while these sets are always the same topologically, the dynamical behavior on them is often quite different. We shall also describe a number of interesting topological objects that arise in the parameter plane (the $\lambda$-plane) for these maps. These include Mandelpinski necklaces, Cantor webs, and Cantor sets of circles of Sierpiński curve Julia sets.
\end{abstract}

\section{INTRODUCTION}

The goal of this paper is to give an overview of some of the many recent results involving singular perturbations of complex dynamical systems. Roughly speaking, a singular perturbation arises when a dynamical system that depends smoothly on a parameter $\lambda$ has the property that, when $\lambda=0$, the system is more or less well understood, but whenever $\lambda \neq 0$, the system becomes much more complicated. In the cases we shall consider, when $\lambda$ becomes nonzero, the degree of the system suddenly becomes much higher. As a consequence, the corresponding dynamical behavior changes dramatically.

Singular perturbations arise in all areas of dynamical systems: ODEs, PDEs, and discrete dynamical systems. See [37, 39, and 63. for numerous examples of singular perturbations in these areas. For a simple example, consider Newton's method applied to the complex polynomial equation $P_{\lambda}(z)=z^{2}-\lambda=0$. (Chances are you would not use Newton's method to solve this equation, but....) The Newton iteration function is then given by

$$
N_{\lambda}(z)=z-\frac{P_{\lambda}(z)}{P_{\lambda}^{\prime}(z)}=z-\frac{z^{2}-\lambda}{2 z}=\frac{z}{2}+\frac{\lambda}{2 z} .
$$

When $\lambda=0$, to find the roots of $P_{0}$, we iterate $N_{0}(z)=z / 2$ and, of course, all orbits of $N_{0}$ tend to the unique root at 0 . But, when $\lambda \neq 0$, the degree of $N_{\lambda}$ jumps from 1 to 2 , and the behavior of the map is quite different. Most orbits of $N_{\lambda}$ still do

Received by the editors June 12, 2012.

2010 Mathematics Subject Classification. Primary 37F10; Secondary 37F45.

The authors work was partially supported by grant \#208780 from the Simons Foundation. 
converge to one of the two roots of $P_{\lambda}$, namely $\pm \sqrt{\lambda}$, but points on the straight line passing through the origin perpendicular to the line segment connecting $\pm \sqrt{\lambda}$ have orbits that do not converge to these roots. Rather, all orbits on this line behave chaotically, so the dynamical behavior is quite different in this case. More generally, whenever Newton's method is applied to a polynomial equation $P_{\lambda}(z)=0$, where $P_{0}$ has a multiple root but $P_{\lambda}$ does not when $\lambda \neq 0$, we find a similar abrupt change in the dynamical behavior 60 .

In this paper, we shall describe the interesting geometry, topology, and dynamics that arise when a complex polynomial is singularly perturbed by adding a pole. For simplicity, we shall consider the dynamics of maps of the form

$$
F_{\lambda}(z)=P(z)+\frac{\lambda}{(z-a)^{d}},
$$

where $\lambda, a \in \mathbb{C}$ and $P$ is a complex polynomial of degree $n \geq 2$ whose dynamics are completely understood. This is a singular perturbation of $P(z)$ since $F_{0}$ is a polynomial of degree $n$, whereas $F_{\lambda}$ is a rational map of degree $n+d$ when $\lambda \neq 0$. Thus we are considering certain subsets of the set of all rational maps of degree $n+d$. But when $\lambda=0$, we reach the boundary of this set, the structure of which has become an important topic in contemporary research [12.

In complex dynamics, the most important object in the dynamical plane is the Julia set. This is the set of points on which the dynamical behavior is chaotic. When a singular perturbation is introduced, this often causes the Julia sets to change dramatically. For example, for the simple function $z^{2}$, the Julia set is known to be the unit circle. But when we singularly perturb this map to one of the form

$$
F_{\lambda}(z)=z^{2}+\frac{\lambda}{z^{2}}
$$

the structure of the Julia set is quite very different; see Figure 1.
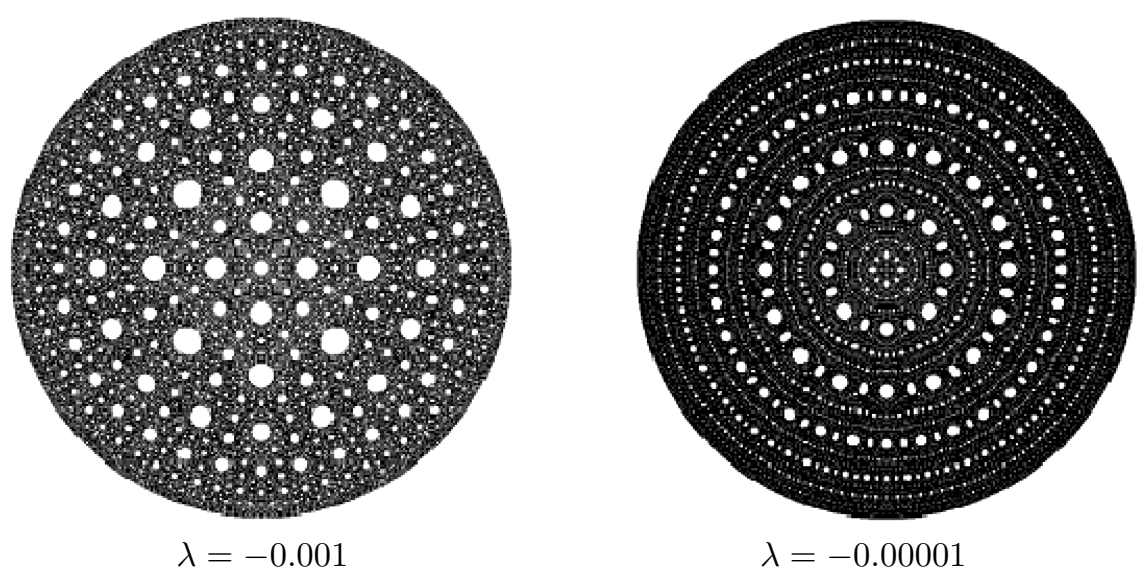

Figure 1. The black points lie in the Julia sets for $z^{2}+\lambda / z^{2}$ where $\lambda=-0.001$ and $\lambda=-0.00001$. When $\lambda=0$, the Julia set is just the unit circle which is very close to the outer boundaries of these two Julia sets. 
It turns out that by far the most interesting types of singular perturbations occur when the pole $a$ is placed at a critical point of $P$ which is also a periodic point, i.e., $a$ lies on a superattracting periodic orbit of $P$. When, for example, the pole is placed in the basin of attraction of this periodic orbit, the resulting behavior is much simpler; see [43, 44]. For this reason we shall concentrate at the outset on the simplest case where $P(z)=z^{n}$ and $a=0$, i.e., the family of maps

$$
F_{\lambda}(z)=z^{n}+\frac{\lambda}{z^{d}}
$$

where $n \geq 2$ and $d \geq 1$. In this case 0 is the only finite critical point of $z^{n}$, and this point is fixed. But when $\lambda \neq 0$, this point suddenly becomes a pole, and we see the kind of exposion in the Julia set illustrated in Figure 1, Later we shall delve more deeply into the case where the polynomial is $P(z)=z^{n}+c$, where $c$ is chosen at the center of a hyperbolic component of the Multibrot set (to be defined below). These are exactly the $c$ values that yield superattracting periodic points for $P$.

Curiously, we shall see in Section 9 that the most difficult (and also the most interesting) case occurs when $n=d=2$, i.e., the family

$$
F_{\lambda}(z)=z^{2}+\frac{\lambda}{z^{2}}
$$

The family

$$
G_{\lambda}(z)=z^{n}+\frac{\lambda}{z}
$$

is also quite different. However, when $n, d \geq 2$ (but not both equal to 2 ), the situation is much easier to understand, at least when $|\lambda| \neq 0$ is small.

One subtheme of this paper will be how various interesting topological objects appear in both the dynamical plane and the parameter plane (the $\lambda$-plane). These include Sierpiński curves and gaskets, Cantor webs and necklaces, and Mandelpinski necklaces, among many others. As is common in the field of complex dynamics, we shall often first observe these topological objects in the dynamical plane and then use ideas from complex analysis to create replications of them in the parameter plane.

\section{THE ESCAPE TRICHOTOMY}

For simplicity, we begin by considering the family of maps

$$
F_{\lambda}(z)=z^{n}+\frac{\lambda}{z^{n}}
$$

where $\lambda \in \mathbb{C}$ and $n \geq 2$. The goal is to understand what happens when we iterate $F_{\lambda}$. So let $F_{\lambda}^{k}$ denote the $k$ th iterate of $F_{\lambda}$. The orbit of $z \in \mathbb{C}$ is the sequence $\left\{F_{\lambda}^{k}(z)\right\}$, where $k=0,1,2, \ldots$ The goal is to understand the fate of all orbits of $F_{\lambda}$.

There are two types of orbits that are very important, the escaping orbits and the periodic orbits or cycles. For $F_{\lambda}$, note that, when $|z|$ is large, we have $\left|F_{\lambda}(z)\right|>|z|$, so orbits of these points tend to $\infty$. The set of all points whose orbits escape to $\infty$ is then called the full basin of attraction of $\infty$. On the other hand, the orbit of $z$ is periodic if $F_{\lambda}^{k}(z)=z$ for some $k>0$. Such a periodic orbit is attracting if $\left|\left(F^{k}\right)^{\prime}(z)\right|<1$, repelling if $\left|\left(F^{k}\right)^{\prime}(z)\right|>1$, and neutral if $\left|\left(F^{k}\right)^{\prime}(z)\right|=1$. If $z$ lies on an attracting periodic orbit, then nearby orbits tend to the orbit of $z$. If $z$ lies on a repelling cycle, then nearby orbits move away from this orbit, at 
least initially. If the cycle is neutral, then the nearby behavior is quite different depending on the argument of $\left(F_{\lambda}^{k}\right)^{\prime}(z)$ and, in certain cases, the nearby behavior is still not completely understood. In fact, one of the major open questions in complex dynamics is to explain the behavior of orbits near a fixed point $z$ for which $F_{0}^{\prime}(z)=\exp (2 \pi i \theta)$, where $\theta$ is an irrational number with certain specific properties [47. Much current research has dealt with this problem [8], 65.

The most important object in the dynamical plane is the Julia set of $F_{\lambda}$; this set has the following two equivalent characterizations.

Definition. $J\left(F_{\lambda}\right)$ is the closure of the set of repelling periodic points. Equivalently, the Julia set is also the boundary of the full basin of $\infty$.

For a proof of the equivalence of these two definitions, see [47]. One consequence of this equivalence is that $J\left(F_{\lambda}\right)$ is a completely invariant set, i.e., both $F_{\lambda}$ and $F_{\lambda}^{-1}$ preserve $J\left(F_{\lambda}\right)$. Another consequence is that $F_{\lambda}$ behaves chaotically on its Julia set, for in any neighborhood of a point $z \in J\left(F_{\lambda}\right)$, there are points whose orbits escape to $\infty$ and other points that lie on a periodic orbit. These very different behaviors indicate that $F_{\lambda}$ has sensitive dependence on intial conditions on $J\left(F_{\lambda}\right)$, the hallmark of chaotic behavior. The complement of the Julia set is called the Fatou set. This is where the dynamical behavior is relatively tame [2], 477, [59].

As a simple example of this, when $\lambda=0$, we have $F_{0}(z)=z^{n}$ and the dynamical behavior of $F_{0}$ is well understood. If $|z|<1$, then the orbit of $z$ tends to the attracting fixed point at 0 . If $|z|>1$, then the orbit of $z$ tends to $\infty$. But if $|z|=1$, then the orbit of $z$ remains forever on the unit circle $S^{1}$. On this circle, the map is given by $\theta \mapsto n \theta$ with the angle $\theta$ defined mod $2 \pi$. Then this circle map is easily seen to have a dense set of periodic points on the circle, namely any angle $\theta=2 \pi p /\left(n^{k}-1\right)$, where $p \in \mathbb{Z}$. These periodic points are all repelling since $\left|F_{\lambda}^{\prime}\right|>1$ on $S^{1}$. So we have that $S^{1}$ is the boundary of the full basin of $\infty$ and also the closure of the set of repelling periodic points. Hence $J\left(F_{0}\right)=S^{1}$. Thus, in the Fatou set, all orbits simply tend to the fixed points at 0 or at $\infty$.

In complex dynamics, it is the orbit of the critical points that often determines the topological structure of the Julia set. For example, consider the family $P_{c}(z)=$ $z^{n}+c$ with $n \geq 2$. The only critical point for $P_{c}$ is 0 , and a classical result due to Fatou 32 in 1906 says that there is an "escape dichotomy":

Theorem. If $P_{c}^{k}(0) \rightarrow \infty$, then $J\left(P_{c}\right)$ is a Cantor set. But if $P_{c}^{k}(0) \not \rightarrow \infty$, then $J\left(P_{c}\right)$ is a connected set.

That is, there are only two topological types of Julia sets for polynomials of the form $z^{n}+c$ : either they are connected sets or else they consist of uncountably many distinct point components. For a proof we refer to [47.

When $n=2$, this dichotomy defines the Mandelbrot set in the parameter plane for $z^{2}+c$ :

Definition. For $z^{2}+c$, the Mandelbrot set is the set of all complex parameters $c$ for which the orbit of the critical point does not escape to $\infty$. Equivalently, the Mandelbrot set is the set of all $c$-values for which the Julia set of $P_{c}$ is a connected set. The analogous set for $z^{n}+c$ is called the Multibrot set.

Our goal in this section is to begin to describe the analogue of the Multibrot set for the family $F_{\lambda}$. At first this would seem complicated because one checks easily that there are $2 n$ free critical points for $F_{\lambda}$ that are given by $c_{\lambda}=\lambda^{1 / 2 n}$. (We call 
these points free critical points because $\infty$ is also a critical point, but this point is always a fixed point in the Riemann sphere, and 0 is another critical point, but the orbit of 0 always lands on $\infty$ after one iteration.)

However, there are only two free critical values for $F_{\lambda}$ given by $v_{\lambda}= \pm 2 \sqrt{\lambda}$ since $n$ of the critical points map to $+2 \sqrt{\lambda}$ and the other $n$ map to $-2 \sqrt{\lambda}$. Moreover, just as in the case of $z^{n}+c$, there really is only one free critical orbit up to symmetry since, when $n$ is odd, $F_{\lambda}^{k}(2 \sqrt{\lambda})=-F_{\lambda}^{k}(-2 \sqrt{\lambda})$, so the critical orbits are symmetric under $z \mapsto-z$. When $n$ is even, we have $F_{\lambda}(2 \sqrt{\lambda})=F_{\lambda}(-2 \sqrt{\lambda})$, so all of the free critical points land on the same orbit after two iterations in this case.

For the family $F_{\lambda}$, the point at $\infty$ is always an attracting fixed point, so we have an immediate basin of attraction at $\infty$. This is the largest connected open set about $\infty$ in the Riemann sphere $\overline{\mathbb{C}}$ consisting of points whose orbits all tend to $\infty$. We denote this set by $B_{\lambda}$. Since $F_{\lambda}(0)=\infty$, there is an open set containing 0 that is mapped by $F_{\lambda}$ to $B_{\lambda}$. This set may or may not be disjoint from $B_{\lambda}$. If it is disjoint from $B_{\lambda}$, we denote it by $T_{\lambda}$. Note that $F_{\lambda}: B_{\lambda} \rightarrow B_{\lambda}$ is $n$-to-one and so is $F_{\lambda}: T_{\lambda} \rightarrow B_{\lambda}$. We therefore call $T_{\lambda}$ the "trap door" since the orbit of any point that eventually enters $B_{\lambda}$ must do so by passing through $T_{\lambda}$. It is also straightforward to check that $F_{\lambda}(\omega z)=-F_{\lambda}(z)$, where $\omega$ is a $2 n$-th root of unity. As a consequence, the orbits of the free critical points all behave symmetrically under $z \mapsto \omega z$. This does not mean that the critical orbits all have the exact same fates; some may tend to one attracting cycle and the others may tend to a different attracting cycle. However, the points on these cycles are all symmetrically located with respect to $z \mapsto \omega z$. Similarly, the sets $J\left(F_{\lambda}\right), B_{\lambda}$, and $T_{\lambda}$ are all symmetric under this rotation, i.e., these sets have $2 n$-fold symmetry.

Unlike the case of $z^{n}+c$, there are three different ways that the critical orbits tend to $\infty$, and this leads to three different types of Julia sets.

Theorem (The escape trichotomy). Suppose the orbits of the free critical points tend to $\infty$.

(1) If $v_{\lambda}$ lies in $B_{\lambda}$, the $J\left(F_{\lambda}\right)$ is a Cantor set.

(2) If $v_{\lambda}$ lies in $T_{\lambda}$, then $J\left(F_{\lambda}\right)$ is a Cantor set of concentric simple closed curves, each one of which surrounds the origin.

(3) In all other cases, $J\left(F_{\lambda}\right)$ is a connected set, and if $F_{\lambda}^{k}\left(v_{\lambda}\right) \in T_{\lambda}$ where $k \geq 1$, then $J\left(F_{\lambda}\right)$ is a Sierpinski curve.

In Figure 2 we display the three different types of Julia sets that arise in the case $n=4$. The black points in these pictures lie in the Julia set; all white points have orbits that tend to $\infty$.

The second part of this result is due to McMullen [45], who showed that this holds as long as $n>2$. For any $n$, it is known that, if $|\lambda|$ is small, the boundary of $B_{\lambda}, \partial B_{\lambda}$, is close to the unit circle (the boundary of the basin of attraction at $\infty$ when $\lambda=0)$. When $n>2$, we have that $F_{\lambda}\left(v_{\lambda}\right)=2^{n} \lambda^{n / 2}+\lambda^{1-n / 2} / 2^{n}$ so that $F_{\lambda}\left(v_{\lambda}\right) \rightarrow \infty$ as $\lambda \rightarrow 0$. Hence $v_{\lambda}$ lies in $T_{\lambda}$ for $\lambda$ small when $n>2$. But when $n=2$ this computation shows that $F_{\lambda}\left(v_{\lambda}\right)=1 / 4+4 \lambda$. So when $\lambda$ is close to 0 , $F_{\lambda}\left(v_{\lambda}\right)$ lies well inside the unit circle and hence $v_{\lambda}$ does not lie in $T_{\lambda}$.

A Sierpinski curve is a planar set that is homeomorphic to the well-known Sierpiński carpet fractal shown in Figure 3. Sierpiński curves are important sets for a number of reasons. First, as we shall show, these sets arise in many different settings in this family of maps. Second, the Sierpinski carpet is a universal plane 

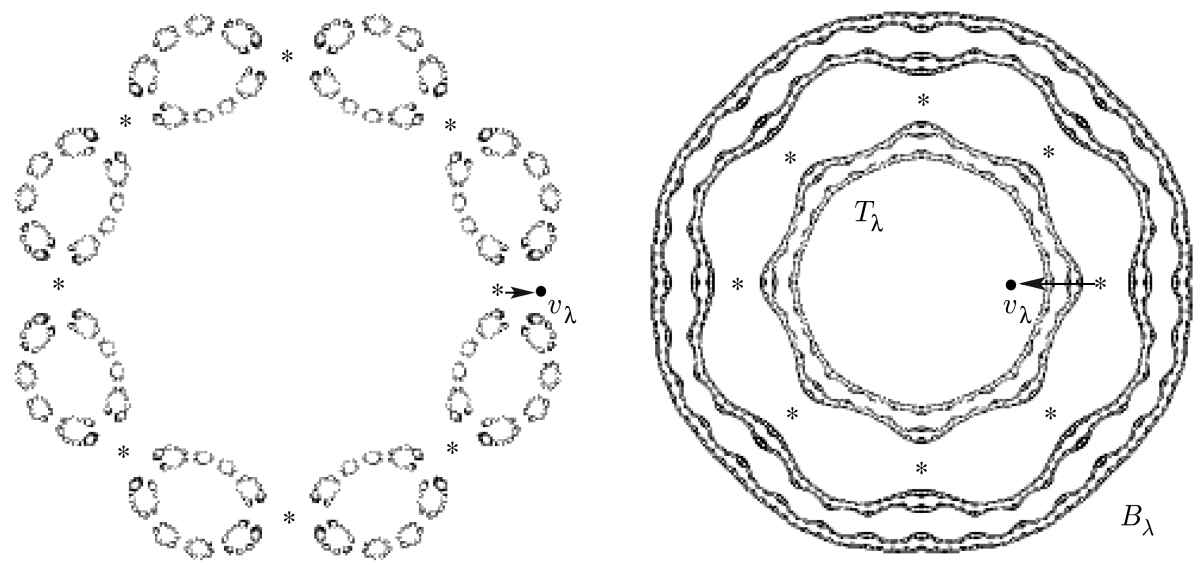

$$
\lambda=0.2
$$

$$
\lambda=0.04
$$

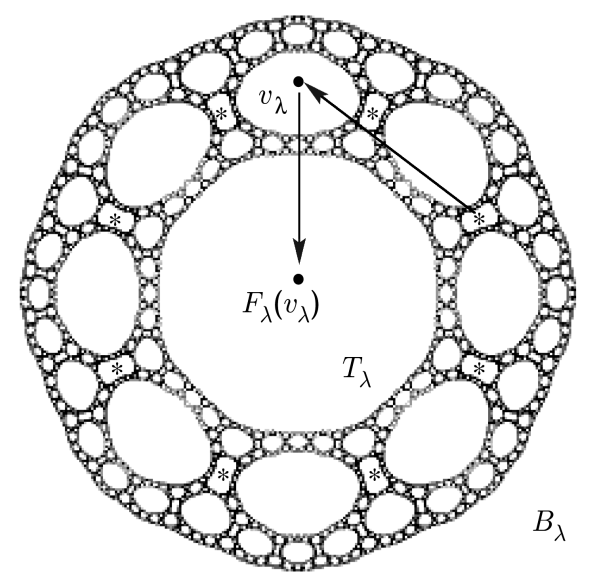

$$
\lambda=-0.1
$$

Figure 2. Some Julia sets for $z^{4}+\lambda / z^{4}$ : if $\lambda=0.2, J\left(F_{\lambda}\right)$ is a Cantor set; if $\lambda=0.04, J\left(F_{\lambda}\right)$ is a Cantor set of circles; and if $\lambda=-0.1, J\left(F_{\lambda}\right)$ is a Sierpiński curve. Asterisks indicate the location of critical points.

continuum in the sense that it contains a homeomorphic copy of every compact, connected, one-dimensional planar set, no matter how intricate this set is. And third, there is a topological characterization of these sets due to Whyburn 64. Any planar set that is compact, connected, nowhere dense, locally connected, and has the property that any two complementary domains are bounded by pairwise disjoint simple closed curves is homeomorphic to the carpet. Four of these properties are usually easy to show for Julia sets using techniques from complex dynamics. First, Julia sets are always compact subsets of the Riemann sphere. Second, it is easy to check that $B_{\lambda}$ and $T_{\lambda}$ are disjoint open disks in the Fatou set whenever the orbits of $\pm v_{\lambda}$ eventually escape, and so all of their preimages are also disjoint open 


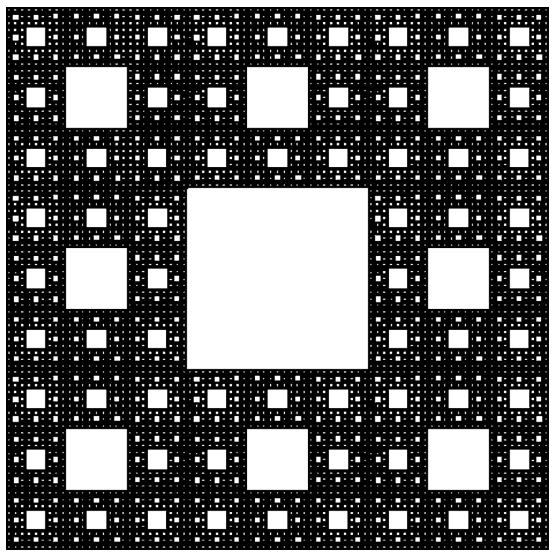

FiguRE 3. The Sierpiński carpet.

disks. A major fact in complex dynamics is that there is always a critical point associated to any periodic Fatou component [4], and the Sullivan No-WanderingDomains Theorem [59] asserts that there can never be a Fatou component that is not (eventually) periodic. Since, in our case, all of the critical orbits escape, it follows that the preimages of $B_{\lambda}$ must make up the entire Fatou set, so the Julia set is connected. Third, it is well known that either a Julia set is nowhere dense or else it is the entire Riemann sphere; in our case, it cannot be the entire sphere since we have the basin at $\infty$, which lies in the Fatou set. And, fourth, a result of Mañé, Sad, and Sullivan 42 says that, if the postcritical set (i.e., the closure of the union of all forward images of the critical points) is disjoint from the Julia set, then the Julia set is locally connected. This happens for $F_{\lambda}$ since we have that all of the critical orbits escape to $\infty$. So all that is necessary to prove is that the boundaries of the Fatou components are disjoint simple closed curves. This was shown in 23.

The proof that the Julia set is a Cantor set when the critical values lie in $B_{\lambda}$ is essentially the same as in the case $z^{n}+c$. It is straightforward to find $2 n$ disjoint, symmetrically located disks $U_{1}, \ldots, U_{2 n}$, each of which is mapped univalently onto a much larger disk that properly contains all $2 n$ of the $U_{j}$. So each $U_{j}$ contains a preimage of all of the other disks. Then each of these preimages contains $2 n$ prepreimages of the preimages of the $U_{k}$ that lie in a given disk. Standard arguments from complex dynamics then show that the set of points whose orbits remain for all iterations in the union of the $U_{k}$ is a Cantor set.

When both critical values lie in $T_{\lambda}$, McMullen's proof proceeds along these lines. Since we are assuming that $B_{\lambda}$ and $T_{\lambda}$ are disjoint, the question is, What is the preimage of $T_{\lambda}$ ? One might at first think that the preimage of the disk $T_{\lambda}$ would be $2 n$ distinct disks, each of which contains one of the critical points. But this cannot happen because each of these disks would then be mapped two-to-one onto $T_{\lambda}$, so there would be $4 n$ preimages of each point in $T_{\lambda}$. But $F_{\lambda}$ has degree $2 n$, so this is not possible. So some of the preimages containing critical points must overlap, but then, by the $2 n$-fold symmetry, all of the preimages of $T_{\lambda}$ must intersect. That is, the preimage of $T_{\lambda}$ is a connected set. Then the Riemann-Hurwitz formula from complex analysis says that $F_{\lambda}^{-1}\left(T_{\lambda}\right)$ is an annulus that is mapped $2 n$-to-one onto $T_{\lambda}$. It then follows easily that the second preimage of $T_{\lambda}$ is a pair of disjoint 

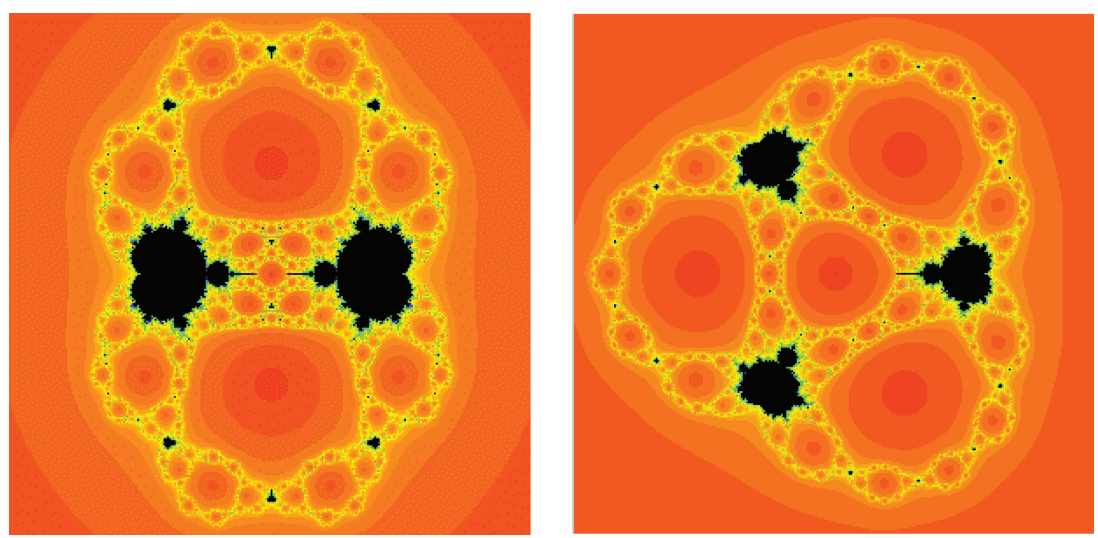

Figure 4 . The parameter planes when $n=3$ and $n=4$.

annuli, each of which is mapped as an $n$-to-one covering onto $F_{\lambda}^{-1}\left(T_{\lambda}\right)$; the third preimage consists of four disjoint annuli similarly mapped to $F_{\lambda}^{-2}\left(T_{\lambda}\right)$; an so on. What McMullen showed was that, removing all of these annuli in the Fatou set leaves behind a Cantor set of simple closed curves, which then forms the Julia set.

Since there is only one critical orbit (up to symmetry) for maps in the family $F_{\lambda}$, the $\lambda$-plane is the natural parameter plane, just as in the case of $z^{n}+c$. In Figure 4, we display the parameter planes (the $\lambda$-planes) for $n=3$ and $n=4$. The external red region in each case consists of parameters for which $v_{\lambda}$ lies in $B_{\lambda}$ so the Julia sets for these parameters are all Cantor sets. This region is the Cantor set locus. The small central red disk surrounding the origin consists of parameters for which $v_{\lambda}$ lies in $T_{\lambda}$, so the Julia sets for parameters drawn from this region are Cantor sets of concentric simple closed curves. This region is called the McMullen domain. It is known that the Julia sets corresponding to all other parameters are connected sets 28, so the complement of the Cantor set locus and the McMullen domain is called the connectedness locus. All of the other red disks in the connectedness locus consist of parameters for which the critical orbit eventually escapes, so the Julia sets corresponding to these parameters are Sierpiński curves. These regions in the parameter plane are called Sierpiński holes. In Figure 4 there are also $(n-1)$ large copies of the Mandelbrot set; these are called the principal Mandelbrot sets. Their existence was proved in [15].

One special property of this family of maps is that, unless the Julia set is a Cantor set, the boundary of $B_{\lambda}$ is always a simple closed curve [52]. This contrasts interestingly with the Julia sets drawn from the family $z^{2}+c$. Here the boundary of the basin at $\infty$ is only a simple closed curve when $c$ lies in the main cardioid of the Mandelbrot set; for all other $c$-values the boundary is much different.

\section{Dynamics on Sierpiński CURVES}

As mentioned in the previous section, $J\left(F_{\lambda}\right)$ is a Sierpiński curve whenever the critical orbits eventually land in the immediate basin of $\infty$, i.e., after three or more iterations. As can be seen in Figure 4 , there are a large number of Sierpiński holes in the parameter planes for these maps. We say that such a hole has escape time $\kappa$ if, for each $\lambda$ in the hole, the critical orbits first land in $B_{\lambda}$ at iteration $\kappa$. A 
parameter $\lambda$ is called the center of the Sierpiński hole if the orbit of the critical points of $F_{\lambda}$ all land on the point at $\infty$ rather than tend to $\infty$. The following result is due to Roesch [54].

Theorem. There is a unique center of each Sierpinski hole. Moreover, there are exactly $(n-1)(2 n)^{\kappa-3}$ Sierpinski holes with escape time $\kappa$ in the parameter plane.

The proof of this result uses quasiconformal surgery techniques [55] to show that there is a unique center of each Sierpiński hole. Given this, the equation for the centers of the holes, namely $F_{\lambda}^{\kappa-1}\left(c_{\lambda}\right)=0$, is easily seen to reduce to a polynomial equation of degree $(n-1)(2 n)^{\kappa-3}$, and so the roots of this equation are all distinct.

As an example of the above count of Sierpinski holes, when $n=3$ there are two Sierpiński holes in the parameter plane with escape time 3; these are the two largest white regions in Figure 4. There are 12 holes with escape time 4, the next largest white holes in that figure. And there are 432 Sierpiński holes with escape time 6 and 120,932,352 holes with escape time 13. All of the parameters from this large collection of Sierpiński holes thus have Julia sets that are homeomorphic, so the natural question is, Are the dynamics on these Julia sets the same?

The answer to this question is given in [27. Two maps $F_{\lambda}$ and $F_{\mu}$ are topologically conjugate on their Julia sets if there is a homeomorphism $h: J\left(F_{\lambda}\right) \rightarrow J\left(F_{\mu}\right)$ that satisfies $F_{\mu} \circ h=h \circ F_{\lambda}$. If two maps are topologically conjugate on their Julia sets, then orbits of $F_{\lambda}$ are mapped to similar orbits of $F_{\mu}$ by $h$, so the maps $F_{\lambda}$ and $F_{\mu}$ essentially have the same dynamical behavior.

Theorem (Escape time conjugacy). Let

$$
F_{\lambda}(z)=z^{n}+\frac{\lambda}{z^{n}} \quad \text { and } \quad F_{\mu}(z)=z^{n}+\frac{\mu}{z^{n}},
$$

where $\lambda$ and $\mu$ are parameters that lie in Sierpinski holes.

(1) If $\lambda$ and $\mu$ lie in the same Sierpinski hole, then $F_{\lambda}$ and $F_{\mu}$ are topologically conjugate on their Julia sets.

(2) If $\lambda$ and $\mu$ lie in Sierpinski holes with different escape times, then $F_{\lambda}$ and $F_{\mu}$ are not topologically conjugate on their Julia sets.

(3) Suppose $\lambda$ and $\mu$ are centers of different Sierpinski holes that have the same escape time. Let $\alpha$ be a primitive $(n-1)$-st root of unity. Then $F_{\lambda}$ and $F_{\mu}$ are topologically conjugate on their Julia sets if and only if, for some integer $j$, either

- $\mu=\alpha^{2 j} \lambda$ or

- $\mu=\alpha^{2 j} \bar{\lambda}$.

Therefore, if $\lambda$ and $\mu$ are parameters that lie in different Sierpinski holes whose escape times are the same, then $F_{\lambda}$ and $F_{\mu}$ are topologically conjugate on their Julia sets if and only if the parameters corresponding to the centers of these Sierpinski holes are symmetrically located with respect to rotation by $\alpha^{2 j}$ or by complex conjugation followed by such a rotation.

The proof of the first part of this theorem follows again by quasiconformal surgery techniques. The second part follows from the fact that any conjugacy between $F_{\lambda}$ and $F_{\mu}$ must take $\partial B_{\lambda}$ to $\partial B_{\mu}, \partial T_{\lambda}$ to $\partial T_{\mu}$, and the $k$ th preimages of $\partial T_{\lambda}$ to the corresponding preimages of $\partial T_{\mu}$. But the preimages of $T_{\lambda}$ and $T_{\mu}$ that contain the critical points are special: their boundaries are mapped two-to-one onto their images, and these are the only preimages of $\partial T_{\lambda}$ and $\partial T_{\mu}$ that have this property. 
Hence, two such conjugate maps must have the same escape times. Finally, for part three, it suffices to consider only maps that are the centers of the corresponding holes. But these maps are "critically finite" in the sense that all of the critical orbits eventually land on the fixed point at $\infty$. By Thurston's Theorem [30, 62, in the orientation preserving case, two such maps can be globally conjugated by a Möbius transformation. But such a conjugacy must then take $\infty$ to $\infty$ (since $\infty$ is the only superattracting fixed point) and 0 to 0 (since 0 is the only preimage of $\infty)$. It follows that the conjugacy must be of the form $z \mapsto \alpha z$ for some $\alpha \in \mathbb{C}$. Then, comparing coefficients in the conjugacy equation,

$$
\alpha F_{\lambda}(z)=F_{\mu}(\alpha z)
$$

shows that $\alpha^{n-1}=1$ and $\mu=\alpha^{2} \lambda$. In the case of an orientation reversing conjugacy, it is easy to check that $F_{\lambda}$ is conjugate to $F_{\bar{\lambda}}$ via $z \mapsto \bar{z}$, so this gives all of the possible conjugate centers of Sierpiński holes.

This result allows us to give a precise count of the number of different conjugacy classes of escape time Sierpiński curves, because only those holes that are symmetric under rotation by successive squares of a primitive $(n-1)$-st root of unity or by complex conjugation have the same dynamics.

Theorem (Number of conjugacy classes). The number of topological conjugacy classes of escape time Sierpiński curve Julia sets with escape time $\kappa$ is given by

(1) $(2 n)^{\kappa-3}$ if $n$ is odd,

(2) $(2 n)^{\kappa-3} / 2+2^{\kappa-4}$ if $n$ is even.

For example, when $n=3$, we have seen that there are exactly 432 Sierpiński holes in this family with escape time 6 , so there are exactly 216 different conjugacy classes of such maps. Similarly, there are 120,932,352 Sierpinski holes with escape time 13 and exactly 60,466, 176 different conjugacy classes, so clearly there is a great variety of different dynamical behaviors on these escape time Sierpiński curve Julia sets. Recently, Moreno Rocha [50] has given a combinatorial invariant which explains why maps drawn from different Sierpiński holes have nonconjugate dynamics.

The reason for the different number of conjugacy classes when $n$ is even and odd comes from the fact that, when $n$ is odd, there are no Sierpinski holes that meet the real axis (and so have no complex conjugate holes that differ from those that are symmetric under the $\alpha^{2}$ rotations). In Figure 4, we see that, along the real axis when $n=3$, there is only a pair of Mandelbrot sets and the McMullen domain. This happens whenever $n$ is odd. As a consequence, there are always exactly $(n-1)$ different Sierpiński holes with conjugate dynamics. When $n$ is even, the situation is very different; there is always a Cantor necklace along the negative real axis and this necklace contains infinitely many Sierpiński holes (more about the Cantor necklace in Section 5). In Figure 4 one can see that there are many Sierpiński holes lying along $\mathbb{R}^{-}$when $n=4$. It turns out that there are many other ways that Sierpiński curves arise as Julia sets in the family $F_{\lambda}$, but before describing this, we turn to some structures in the parameter plane.

\section{MANDELPinski NECKLACES}

As we have seen, when $n \geq 3$ the origin in the parameter plane is surrounded by the McMullen domain which we denote by $\mathcal{M}$. All parameters in this domain have Julia sets that are Cantor sets of simple closed curves surrounding the origin. It 
is known [16] that the McMullen domain is a disk surrounding the origin, but this disk in the parameter plane is surrounded by much more structure.

In Figure 5 we display two magnifications of the parameter plane around $\mathcal{M}$ when $n=3$. Note that there appears to be a collection of rings surrounding the boundary of $\mathcal{M}$ that pass through more and more Sierpiński holes (the white regions) as these rings approach $\partial \mathcal{M}$. Closer inspection seems to indicate that these rings also pass through small copies of Mandelbrot sets as well. This is indeed true, as the following result was shown in [16] and [24.

Theorem (Rings around the McMullen domain). For each $n \geq 3$, the McMullen domain is surrounded by infinitely many "Mandelpinski necklaces" $\mathcal{S}^{k}$ for $k=$ $1,2, \ldots$ These are simple closed curves that have the properties that:

(1) Each curve $\mathcal{S}^{k}$ surrounds $\mathcal{M}$ as well as $\mathcal{S}^{k+1}$, and the $\mathcal{S}^{k}$ accumulate on the boundary of the McMullen domain as $k \rightarrow \infty$.

(2) The curve $\mathcal{S}^{k}$ meets the centers of $\tau_{k}^{n}$ Sierpinski holes, each with escape time $k+2$, where

$$
\tau_{k}^{n}=(n-2) n^{k-1}+1
$$

(3) The curve $\mathcal{S}^{k}$ also passes through $\tau_{k}^{n}$ centers of baby Mandelbrot sets with base period $k$ (when $k \neq 2$ ), and these Mandelbrot sets and Sierpinski holes alternate as the parameter winds around $\mathcal{S}^{k}$.

The one exception to this result is the ring $\mathcal{S}^{2}$. This curve passes through $\tau_{2}^{n}$ centers of Sierpiński holes, but only $\tau_{2}^{n}-(n-1)$ centers of baby Mandelbrot sets. Instead, this curve also passes through the centers of $(n-1)$ period 2 bulbs attached
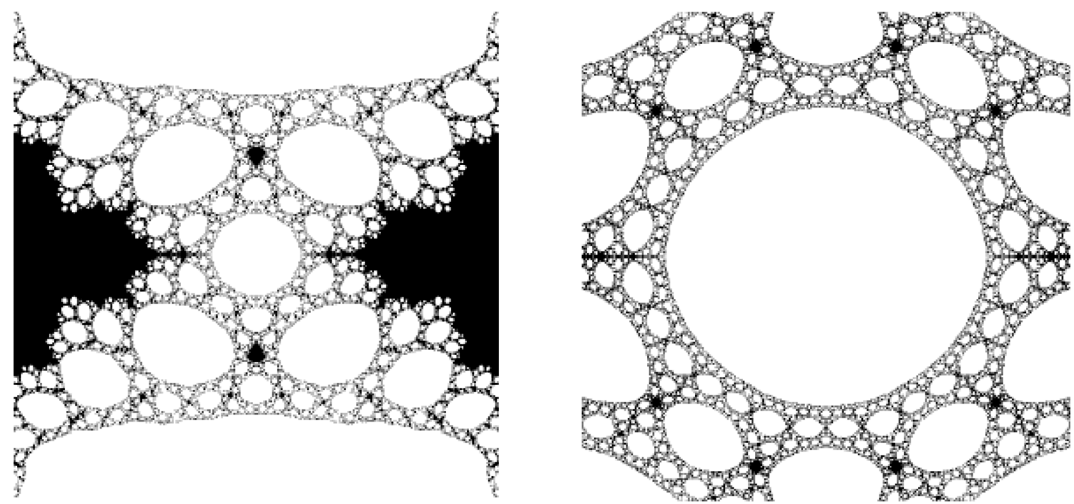

FiguRE 5. Several magnifications of the parameter plane for the family $z^{3}+\lambda / z^{3}$ showing the rings surrounding the McMullen domain $\mathcal{M}$. These rings accumulate on the boundary of $\mathcal{M}$ and contain more and more Sierpiński holes as they do so. 


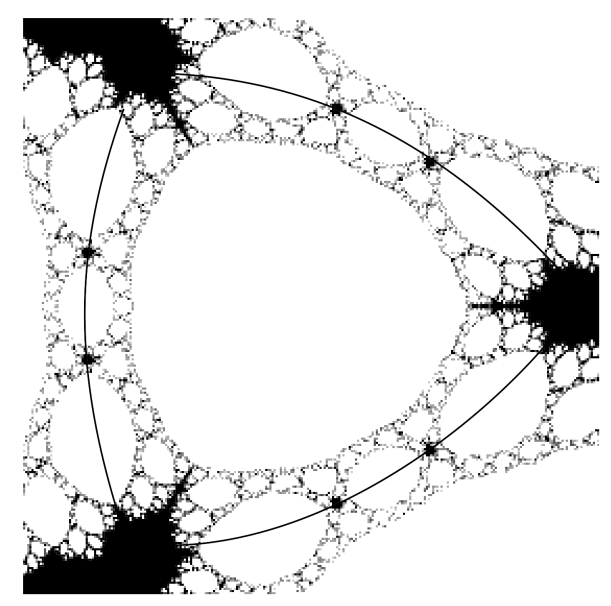

FiguRE 6. The Mandelpinski necklace $\mathcal{S}^{2}$ in parameter plane for $n=4$.

to the main cardioids of the principal Mandelbrot sets. In Figure 6 we display $\mathcal{S}^{2}$ in the case $n=4$. This necklace passes through the centers of six baby Mandelbrot sets and the three centers of the period 2 bulbs of the principal Mandelbrot sets.

By a center of a baby Mandelbrot set with base period $k$, we mean the parameter drawn from the main cardioid of the Mandelbrot set for which the corresponding attracting cycle is actually superattracting, i.e., one of the critical points of $F_{\lambda}$ is periodic. Because there may be two different but symmetric critical orbits when $n$ is odd, the period of these cycles may be $k$ or $2 k$.

The proof of the Rings Theorem is based on the idea is that we first construct similar structures in the dynamical plane and then use techniques from complex analysis to transport them over to the parameter plane. To find the rings in the dynamical plane, recall that we have $2 n$ critical points given by $\lambda^{1 / 2 n}$. There are also $2 n$ prepoles given by $(-\lambda)^{1 / 2 n}$. Note that all of the critical points and prepoles lie on the circle $|z|=|\lambda|^{1 / 2 n}$. This circle is called the critical circle and is denoted by $C_{\lambda}$. A straightforward computation then shows that $C_{\lambda}$ is mapped $2 n$-to-one onto the line segment connecting the two critical values $\pm_{\lambda}$ and passing through the origin. This is the critical line. Furthermore, any other circle centered at the origin is mapped by $F_{\lambda} n$-to-one onto an ellipse whose foci are the critical values. For circles outside (resp., inside) the critical circle, the size of the image ellipse increases as the radius of the circle increases (resp., decreases). Hence $F_{\lambda}$ is an $n$-to-one covering map on both the interior and the exterior of $C_{\lambda}$.

Now when $\lambda$ satisfies $|\lambda|<2^{-2 n /(n-1)}$, one checks easily that $\left|v_{\lambda}\right|<\left|c_{\lambda}\right|$. So the critical circle $C_{\lambda}$ lies in the exterior of its image, the critical line. As a consequence, there is a preimage of $C_{\lambda}, \zeta_{\lambda}^{1}$, that lies outside of $C_{\lambda}$ and that is mapped $n$-to-one onto $C_{\lambda}$. Then there is an outer preimage of $\zeta_{\lambda}^{1}, \zeta_{\lambda}^{2}$, that is mapped $n$-to-one to $\zeta_{\lambda}^{1}$, and so forth. We thus find an infinite collection of closed curves $\zeta_{\lambda}^{k}$ moving outward from the critical circle in the dynamical plane and, because $F_{\lambda}$ is $n$-to-one on each $\zeta_{\lambda}^{k}$, the curve $\zeta_{\lambda}^{k}$ contains exactly $n^{k} \cdot 2 n$ points that are mapped by $F_{\lambda}^{k}$ to one of the critical points in $C_{\lambda}$ and the same number of points that are similarly mapped to prepoles in $C_{\lambda}$. So we have a similar structure in the dynamical plane 
that we wish to prove in the parameter plane, an infinite collection of simple closed curves alternately containing points that are mapped to prepoles and points that are mapped to critical points by $F_{\lambda}^{k}$.

To produce the same picture in the parameter plane, recall that we have assumed that $v_{\lambda}$ lies inside $C_{\lambda}$. The region in the interior of the critical circle is mapped as an $n$-to-one covering of the exterior of the critical line. So one can consider the map $\phi(\lambda)=F_{\lambda}\left(v_{\lambda}\right)$, where $v_{\lambda}$ is a specifically chosen critical value of $F_{\lambda}$. This is a map that takes the parameter plane to the dynamical plane. One checks easily that there is $(n-1)$-fold symmetry in the parameter plane; note the two symmetrically located Mandelbrot sets in the parameter plane for $n=3$ and the three such sets for $n=4$ in Figure 4. One can show that the map $\phi$ is univalent on each of the $(n-1)$ open symmetry sectors in the parameter plane bounded by the straight rays through the "spines" of the $(n-1)$ principal Mandelbrot sets symmetrically arranged around the origin. Moreover, $\phi$ takes each such sector onto $\mathbb{C}$ minus a pair of half-lines which are the images of the spines.

Now consider a particular $k$ th preimage of one of the critical points lying in $C_{\lambda}$ that lies in $\zeta_{\lambda}^{k}$. Call this point $u_{\lambda}$. Then $u_{\lambda}$ varies analytically with $\lambda$ as $\lambda$ ranges over each of the symmetry sectors. So we can consider the analytic map $\Phi(\lambda)=\phi^{-1}\left(u_{\lambda}\right)$. This map takes the sector in the parameter plane to itself. Then one can show using the Schwarz Lemma that $\Phi$ has a unique fixed point in this sector. This fixed point is a parameter $\lambda^{*}$ for which $\phi\left(\lambda^{*}\right)=u_{\lambda^{*}}$, i.e., $F_{\lambda^{*}}\left(v_{\lambda^{*}}\right)$ lands on the given $k$ th preimage of a critical point. Then this critical point is fixed by $F_{\lambda^{*}}^{k+2}$ if $n$ is even or is either fixed or has period 2 under $F_{\lambda^{*}}^{k+2}$ if $n$ is odd (because of the $z \mapsto-z$ symmetry). This produces a center of a baby Mandelbrot set for each of the given critical points on $\zeta_{\lambda}^{k}$ (modulo an identification as $\lambda$ winds around the origin). We similarly get centers of Sierpiński holes by letting $u_{\lambda}$ be a preimage of a prepole instead of a critical point.

This produces the centers of Sierpiński holes and baby Mandelbrot sets in the parameter plane. One can produce the entire Mandelpinski necklace by constructing a natural parametrization of $C_{\lambda}$, pulling it back to $\zeta_{\lambda}^{k}$, and then using the Schwarz Lemma as above where now $u_{\lambda}=u_{\lambda}(\theta)$ is a particular point on the parametrization of $\zeta_{\lambda}^{k}(\theta)$. The fact that we actually get a Sierpiński hole surrounding the center produced above follows from Roesch's result [54, while the existence of the complete baby Mandelbrot set uses polynomial-like map arguments 31. See [14 for details.

\section{Cantor necklaces And Webs}

In this section, we describe some different types of topological structures known as Cantor necklaces and webs that appear in both the dynamical and parameter planes for $F_{\lambda}$. We will begin with the simplest case involving Cantor necklaces that arise in the family $z^{2}+\lambda / z^{2}$. Later we will describe a more general topological structure, the Cantor web, that appears in the families of maps with $n \geq 3$.

First we construct a model Cantor necklace, namely the Cantor middle-thirds necklace $N$. Here we start with the Cantor middle-thirds set along the positive real axis in the plane. Then we adjoin an open disk of diameter $1 / 3^{k}$ in place of every open interval of length $1 / 3^{k}$ that has been removed. The resulting set is the Cantor middle-thirds necklace; see Figure 7. Note that $N$ is a connected subset of the plane. A Cantor necklace is then any planar set that is the image of $N$ under a map that is continuous, one-to-one, and onto. 


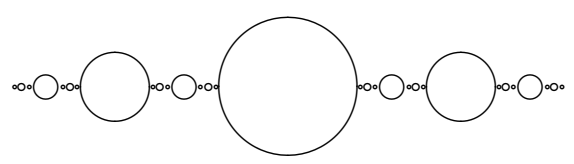

Figure 7. The Cantor middle-thirds necklace.

Now we show how a Cantor necklace arises in the dynamical plane for $z^{2}+\lambda / z^{2}$. Let us assume that $\lambda \notin \mathbb{R}^{+}$, so $\lambda=|\lambda| e^{i \eta}$ where $0<\eta<2 \pi$. The case where $\lambda \in \mathbb{R}^{+}$is similar but has several additional technicalities, so we will avoid this case. Let us also assume that $|\lambda|<1$. If $|\lambda|<1$ and $|z|>2$, we have

$$
\left|F_{\lambda}(z)\right| \geq|z|^{2}-\frac{|\lambda|}{|z|^{2}} \geq 2|z|-\frac{1}{4}>\frac{3}{2}|z| \text {. }
$$

Inductively, it follows that

$$
\left|F_{\lambda}^{k}(z)\right| \geq\left(\frac{3}{2}\right)^{n}|z|,
$$

so any point on or outside the circle of radius 2 centered at the origin lies in $B_{\lambda}$ when $|\lambda|<1$.

Recall that the critical points of $F_{\lambda}$ are given by $\lambda^{1 / 4}$. Therefore one of the critical points of $F_{\lambda}$ lies on the straight line through the origin given by $t \exp (i \eta / 4)$ with $t>0$. We call this a critical line. The image of this line lies along the straight line with argument $\theta=\eta / 2$, and $F_{\lambda}$ maps this critical line in two-to-one fashion over the portion of this straight line that lies beyond the critical value $2 \sqrt{\lambda}$ whose argument is $\eta / 2$. This is a critical value ray. Note that the critical value ray is disjoint from the critical line since we have assumed that $0<\eta<2 \pi$. There is a second critical point of $F_{\lambda}$ lying on the line with argument $\theta=\eta / 4-\pi / 2$, and the corresponding critical line is mapped in two-to-one fashion to the opposite critical value ray lying on $\theta=-\eta / 2$ exactly as above.

Now we know that any point on or outside $r=2$ is mapped closer to $\infty$. Let $\beta_{\lambda}$ denote the image of this circle, so that $\beta_{\lambda} \subset B_{\lambda}$. One checks easily that there is a second circle, namely $r=|\lambda|^{1 / 2} / 2$, that is also mapped two-to-one onto $\beta_{\lambda}$.

Consider the open region $I_{0}=I_{0}^{\lambda}$ bounded by the rays $\theta=\eta / 4$ and $\theta=\eta / 4-\pi / 2$ and the two circular preimages of $\beta_{\lambda}$. The set $I_{0}$ is a quarter of an annulus. Let $I_{1}=-I_{0}$. We call $I_{0}$ (resp., $I_{1}$ ) the right (resp., left) fundamental sector. These fundamental sectors are a pair of disjoint, open, simply connected regions in $\mathbb{C}$. Note that, for each $\lambda, I_{0}$ lies in the right half-plane $\operatorname{Re} z>0$, while $I_{1}$ lies in the left half-plane; see Figure 8 .

By construction, $F_{\lambda}$ maps each of the fundamental sectors in one-to-one fashion onto the open set $\mathcal{O}$ bounded by $\beta_{\lambda}$ minus the portions of the critical value rays extending out to $\beta_{\lambda}$. So the image of each of these fundamental sectors contains the closures of both $I_{0}$ and $I_{1}$ in its interior. Since $F_{\lambda}$ maps the union of the fundamental sectors strictly outside itself, most points in $I_{0} \cup I_{1}$ have orbits that leave this set at some iteration. Let $\Gamma_{\lambda}$ be the set of points whose orbits remain for all iterations in $I_{0} \cup I_{1}$. Then standard arguments from complex dynamics show that $\Gamma_{\lambda}$ is a Cantor set. Moreover, given any infinite sequence $s=\left(s_{0} s_{1} s_{2} \cdots\right)$, where each $s_{j}$ is either 0 or 1 , there is a unique point $z_{s}$ in $\Gamma_{\lambda}$ whose orbit moves 


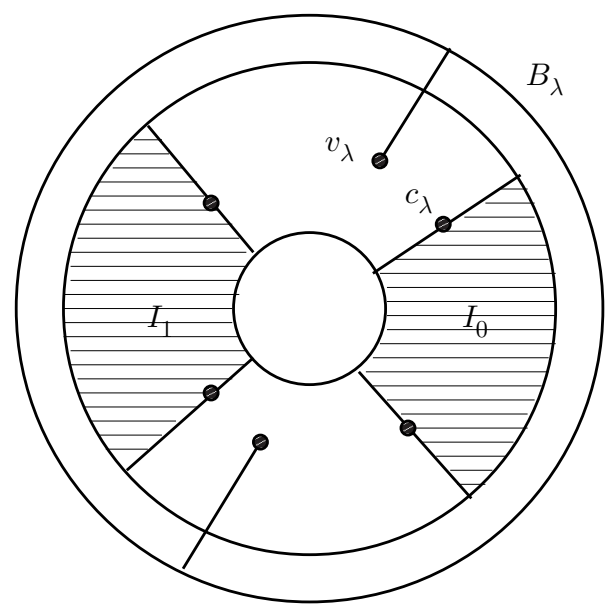

FiguRE 8. $I_{0}$ and $I_{1}$ and their image under $F_{\lambda}$, which is the interior of $\beta_{\lambda}$ minus the two segments connecting this circle to the critical values.

around in $I_{0} \cup I_{1}$ following exactly the pattern given by $s$. The sequence $s$ is called the itinerary of $z_{s}$.

We remark that when $\lambda \in \mathbb{R}^{-}$, the Cantor set $\Gamma_{\lambda}$ lies on the real axis. Indeed, a glance at the graph of the real function $F_{\lambda}$ shows that $F_{\lambda}$ maps the interval $\left[-p_{\lambda}, p_{\lambda}\right]$ in two-to-one fashion over itself, where $p_{\lambda}$ is the fixed point for $F_{\lambda}$ on the positive real axis and on the boundary of $B_{\lambda}$; see Figure 9 ,

Now suppose in addition that the critical values do not lie in $B_{\lambda}$. So, as described in Section $2, J\left(F_{\lambda}\right)$ is a connected set and $B_{\lambda}$ is a simply connected open set. Our goal is to construct a Cantor necklace in the dynamical plane. The Cantor set portion of the necklace will be the set $\Gamma_{\lambda}$ constructed above, whereas the open disks will be certain of the preimages of the basin of $\infty$ lying in $I_{0}$ and $I_{1}$.

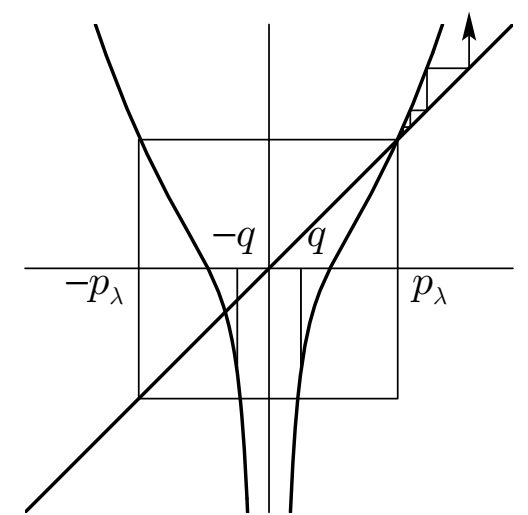

Figure 9. The graph of $F_{\lambda}$ on the real line for $\lambda<0$. The points $\pm q$ bound the trap door on the real axis. 


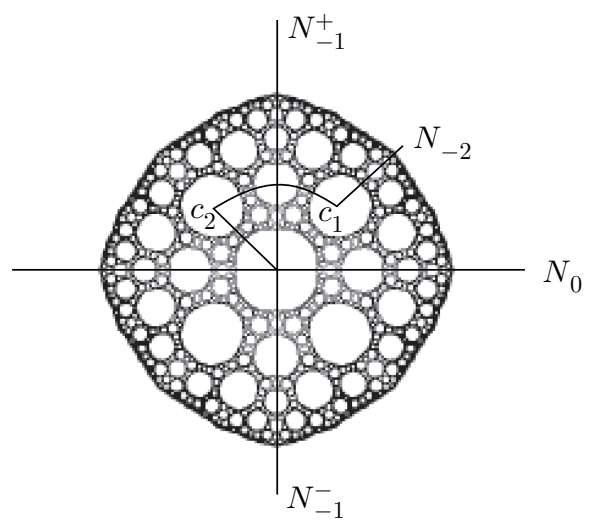

Figure 10. The Julia set for $z^{2}-1 / 16 z^{2}$. The line $N_{0}$ passes through the "almost" invariant Cantor necklace. $F_{\lambda}\left(N_{-1}^{ \pm}\right)=N_{0}$ and so these curves pass through the two preimages of this Cantor necklace. And $F_{\lambda}\left(N_{-2}\right)=N_{-1}^{+} \cup N_{-1}^{-}$. This curve passes through two critical points of $F_{\lambda}$, hence the $90^{\circ}$ turns in this curve.

More precisely, there is a unique fixed point $p_{\lambda}$ lying in $\partial B_{\lambda}$; this is the point whose itinerary is $(111 \cdots)$. The point $-p_{\lambda}$ also lies in $\partial B_{\lambda}$ and has itinerary $(0111 \cdots)$. These are the only two points that lie in $\Gamma_{\lambda} \cap \partial B_{\lambda}$. Let $\pm q_{\lambda}$ be the two preimages of $-p_{\lambda}$ lying in the boundary of the trap door. Since $T_{\lambda}$ is open and simply connected, we may define a homeomorphism that takes a disk centered at $1 / 2$ on the real line and having radius $1 / 6$ onto $T_{\lambda}$. This adjoins the trap door to $\Gamma_{\lambda}$. One checks easily that both critical value rays do not meet $T_{\lambda}$, so there are a pair of preimages of $T_{\lambda}$, one lying in $I_{0}$ and the other lying in $I_{1}$. So we may adjoin these disks to $\Gamma_{\lambda}$ as well. Continuing in this fashion, by choosing only those preimages of $T_{\lambda}$ whose forward orbit lies in $I_{1} \cup I_{0}$, we produce a Cantor necklace in the dynamical plane for $F_{\lambda}$.

Note that this Cantor necklace is "almost" invariant under $F_{\lambda}$. The Cantor set portion is invariant, but only the trap door is mapped outside of the necklace to $B_{\lambda}$. Thus we may produce infinitely many other necklaces by pulling back this necklace by branches of the inverse of $F_{\lambda}$. In Figure 10 we display the Julia set for $\lambda=-1 / 16$. Here the Cantor necklace lies along the real axis and its two other preimages lie along the imaginary axis. In this figure we have drawn curves passing through the Cantor necklaces and several of its preimages. These curves can be thought of as "internal rays". In polynomial dynamics, one of the most important tools is the existence of external rays. Basically, external rays are curves in the basin of $\infty$ that are the images of the straight rays in the unit disk when the Riemann Mapping Theorem is used to uniformize the basin. The fact that these external rays may be extended through the Julia set via Cantor necklaces provides a very different type of tool to understand the structure of these sets; see [10, [52].

To produce an analogous necklace in the parameter plane, we proceed as in the previous section. The Cantor set portion of the necklace arises as follows. Let $D$ be the half-disk in the parameter plane given by $\{\lambda|\operatorname{Re} \lambda<0,| \lambda \mid<1\}$. We have two functions defined on $D$ and taking values in the dynamical plane. Suppose $s$ 
is the itinerary of a point in $\Gamma_{\lambda}$ whose first entry is 0 . Then, for each $\lambda \in D$ there is a unique point $z_{s}(\lambda)$ with that itinerary. Moreover, $z_{s}(\lambda)$ depends analytically on $\lambda$. The second function defined on $D$ is $V(\lambda)=F_{\lambda}\left( \pm v_{\lambda}\right)=4 \lambda+1 / 4$, i.e., the second iterate of the critical points. Note that $V_{\lambda}$ maps $D$ onto a large half-disk that completely contains $I_{1}$ for each $\lambda \in D$. Moreover, $V_{\lambda}$ is invertible. Hence we may consider the composition $V_{\lambda}^{-1} \circ z_{s}(\lambda)$ which takes $D$ strictly inside itself. Again by the Schwarz Lemma, there is a unique fixed point $\lambda_{s}$ for this map. The parameter $\lambda_{s}$ then has the property that the second iterate of the critical point of $F_{\lambda_{s}}$ lies on $z_{s}$. As the itinerary varies over all such itineraries that begin with 0 , this produces a Cantor set of parameters with this property. This is the Cantor set portion of the necklace in parameter space.

For the open regions in the Cantor necklace, we can use the Riemann Mapping Theorem to assign a specifc address to each point in a given preimage of the trap door in the necklace in the dynamical plane together with an itinerary describing how this preimage moves about $I_{1} \cup I_{0}$ under iteration. Then a slightly more complicated argument as above produces the open regions in the Cantor necklace in the parameter plane; see [19] for details. In fact, the Cantor set portion of this Cantor necklace in the parameter plane lies along the negative real axis. This is a consequence of the special nature of the graph of $F_{\lambda}$ (see Figure 9) when $\lambda \in \mathbb{R}^{-}$ together with the fact that the second iterate of the critical points always lies in $\mathbb{R}^{-}$in this case.

In Figure 11] we display the paramter plane for the family $z^{2}+\lambda / z^{2}$ as well as two magnifications. Note that there appear to be many other Cantor necklaces in the parameter plane besides the one along the real axis, just as in the dynamical plane. So the concept of internal rays can also be used to understand the structure of the connectedness locus in the parameter plane.

In the case $n \geq 3$, the Cantor necklaces constructed above are replaced with more complicated objects known as Cantor $(n-1)$-webs. To define the model Cantor $k$-web $S_{k}$ where $k \geq 2$, we begin with the special case $k=2$. Start with the closed unit square in the complex plane. Then remove the open middle-thirds vertical strip $1 / 3<x<2 / 3$ and the open middle-thirds horizontal strip $1 / 3<y<2 / 3$. This leaves behind four closed squares. Then continue this process, at the $j$ th stage removing both the horizontal and vertical middle-thirds strips of lengths $1 / 3^{j}$ from each remaining square. In the limit, the remaining set is a Cantor set $C_{2}$ which is, in fact, the product of a pair of Cantor middle-thirds sets lying on each of the axes. Let $H_{2}$ be the set of all horizontal strips that have been removed. So $H_{2}$ consists of one open rectangle with horizontal length 1 , four open rectangles with horizontal length $1 / 3,4^{2}$ open rectangles with horizontal length $1 / 3^{2}$, etc. Then the set $S_{2}$ is the union of $\mathrm{C}_{2}$ and $\mathrm{H}_{2}$. Note that, on the boundary of each open rectangle in $\mathrm{H}_{2}$ that has been adjoined, there is a Cantor set of points that lie in $C_{2}$. But there are also uncountably many other points in $S_{2}$ that are "buried", i.e., they do not lie on the boundary of one of the open rectangles that have been adjoined.

For the Cantor web $S_{k}$, we begin again with the closed unit square. Now we remove one open horizontal strip given by $1 /(2 k-1)<y<(2 k-2) /(2 k-1)$ and $(k-1)$ open vertical strips whose horizontal length is $1 /(2 k-1)$ so that we are left with $2 k$ closed squares, each with sidelength $1 /(2 k-1), k$ squares along the lower boundary and $k$ along the upper boundary of the unit square. Then continue with this process, each time removing a single open horizontal strip and $(k-1)$ equal 

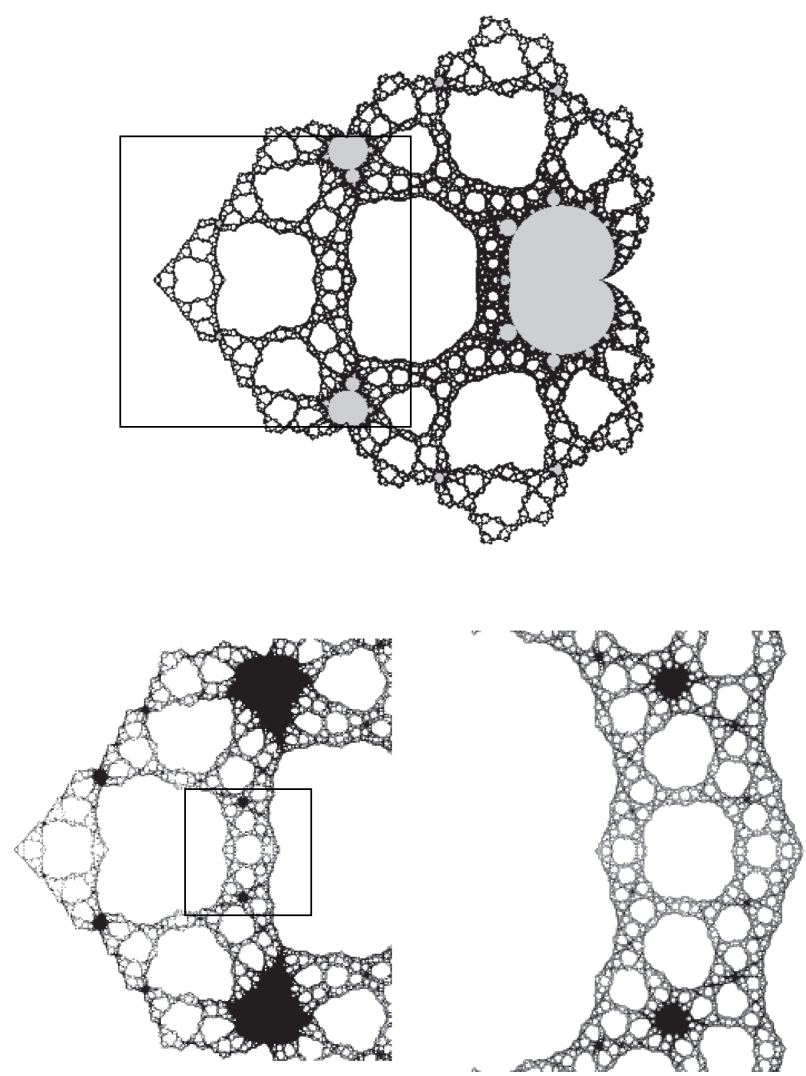

FiguRE 11 . The parameter plane for the family $z^{2}+\lambda / z^{2}$ together with two magnifications. Note the Cantor necklace along the negative real axis.

sized vertical strips from each remaining square so that exactly $2 k$ smaller closed squares remain in each square. In the limit we again get a Cantor set. And, as before, we add back in all of the removed open horizontal strips to get the Cantor web $S_{k}$. A Cantor $k$-web is then any planar set that is the image of $S_{k}$ under a map that is continuous, one-to-one, and onto.

We now sketch a proof that there infinitely many Cantor $(n-1)$-webs in the dynamical plane for the family $z^{n}+\lambda / z^{n}$ when $n \geq 3$. For simplicity we shall deal only with the case $n=3$. Recall that there are six critical points for $F_{\lambda}$ given by $c_{\lambda}=\lambda^{1 / 6}$. Let $c_{0}$ be the critical point whose argument is $\operatorname{Arg} \lambda / 6$ where $0 \leq \operatorname{Arg} \lambda<$ $2 \pi$, and let $c_{1}, \ldots, c_{5}$ be the other critical points arranged in a counterclockwise direction. As earlier, there is a critical line extending from the origin through each of these critical points, and each critical line is mapped two-to-one onto one of the two critical value rays. Let $I_{j}$ denote the sector bounded by the two critical lines passing through $c_{j}$ and $c_{j+1}$. Then, just as in the case where $n=2, F_{\lambda}$ maps the interior of $I_{j}$ univalently onto $\mathbb{C}$ minus the two critical value rays. If $\lambda$ lies in the upper half-plane, then the critical value rays always lie in $I_{0}$ and $I_{3}$. So each of $I_{1}, I_{2}, I_{4}$, and $I_{5}$ are mapped univalently over the union of these four regions. 

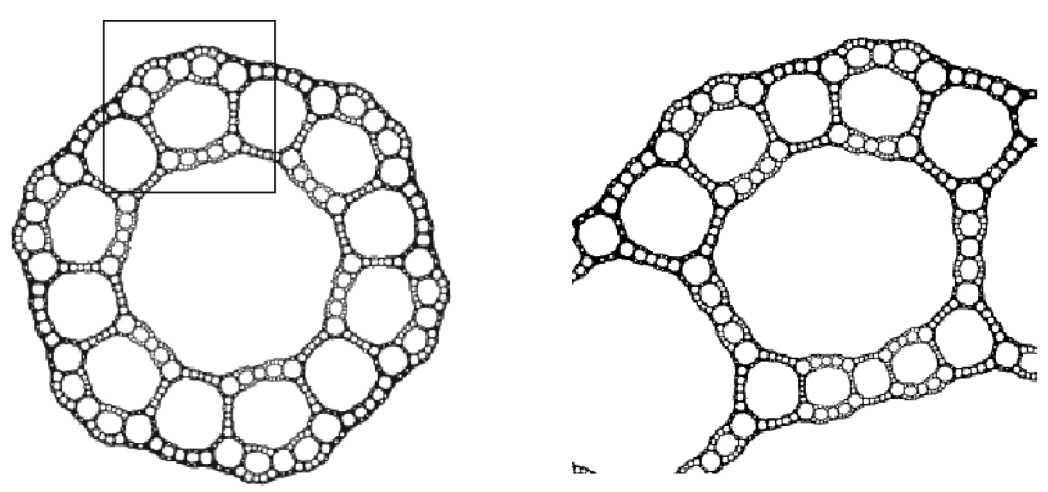

Figure 12. The Julia set of $F_{\lambda}(z)=z^{3}+0.125 / z^{3}$ and a magnification illustrating a Cantor 2-web. While it appears that the central disks are always flanked by more than four smaller disks, we do not include in the construction those disks that map to $I_{0}$ or $I_{3}$ since, for certain parameters, these could contain critical points.

To construct the Cantor 2-web, assume that $\lambda$ lies in the portion of the connectedness locus that lies in the upper half-plane. Therefore $B_{\lambda}$ and $T_{\lambda}$ are disjoint open disks. Then, using similar estimates as in the previous section, we can find a pair of circles, one in $B_{\lambda}$ and one in $T_{\lambda}$, that are mapped well outside the chosen circle in $B_{\lambda}$. Let $W_{j}$ be the portion of the sector $I_{j}$ contained between these two circles. So $W_{j}$ is now a sixth of an annulus. Then consider the union of the four sets $W_{1}, W_{2}, W_{4}$, and $W_{5}$. Call this set $W$. By construction, $F_{\lambda}$ maps each $W_{j}$ in $W$ univalently over all of $W$. Then, just as we showed earlier, standard arguments from complex dynamics again show that the set of points in $W$ whose orbits remain for all time in $W$ is a Cantor set. This is the Cantor set portion of the Cantor 2 -web, and each such point can be assigned an itinerary which is now an infinite sequence whose entries are one of the four integers 1, 2, 4, or 5. Just as in the case of the Cantor necklace, we may adjoin the preimages of the trap door whose orbits remain in the region $W$. To each point in these preimages we can again specify its address by using the Riemann Mapping Theorem together with the itinerary of the preimage as it moves around $W$; see Figure 12 .

To construct the Cantor 2-web in the parameter plane, we proceed in similar fashion as in the Cantor necklace case. First note that the two portions of the sectors $W_{0}$ and $W_{3}$ that we did not consider above are each mapped univalently over the entire set $W$. So there is a preimage of the Cantor 2-web in each of these sets. Now when $\lambda$ lies in the upper half-plane, one critical value always lies in the sector $I_{0}$, the other in $I_{3}$. Thus we again have a univalent map $\lambda \mapsto v_{\lambda}$ that takes a portion of the upper half-plane univalently over the the set $W_{0}$. As before, the Schwarz Lemma then allows us to find a unique parameter for which one of the critical values $v_{\lambda}$ lands on the point in $I_{0}$ and $I_{3}$ with the specified address. This creates a Cantor 2-web in the parameter plane. If we consider other preimages of the Cantor 2-web constructed above, then a similar argument produces other Cantor 2-webs in the parameter plane; see Figure 13. 

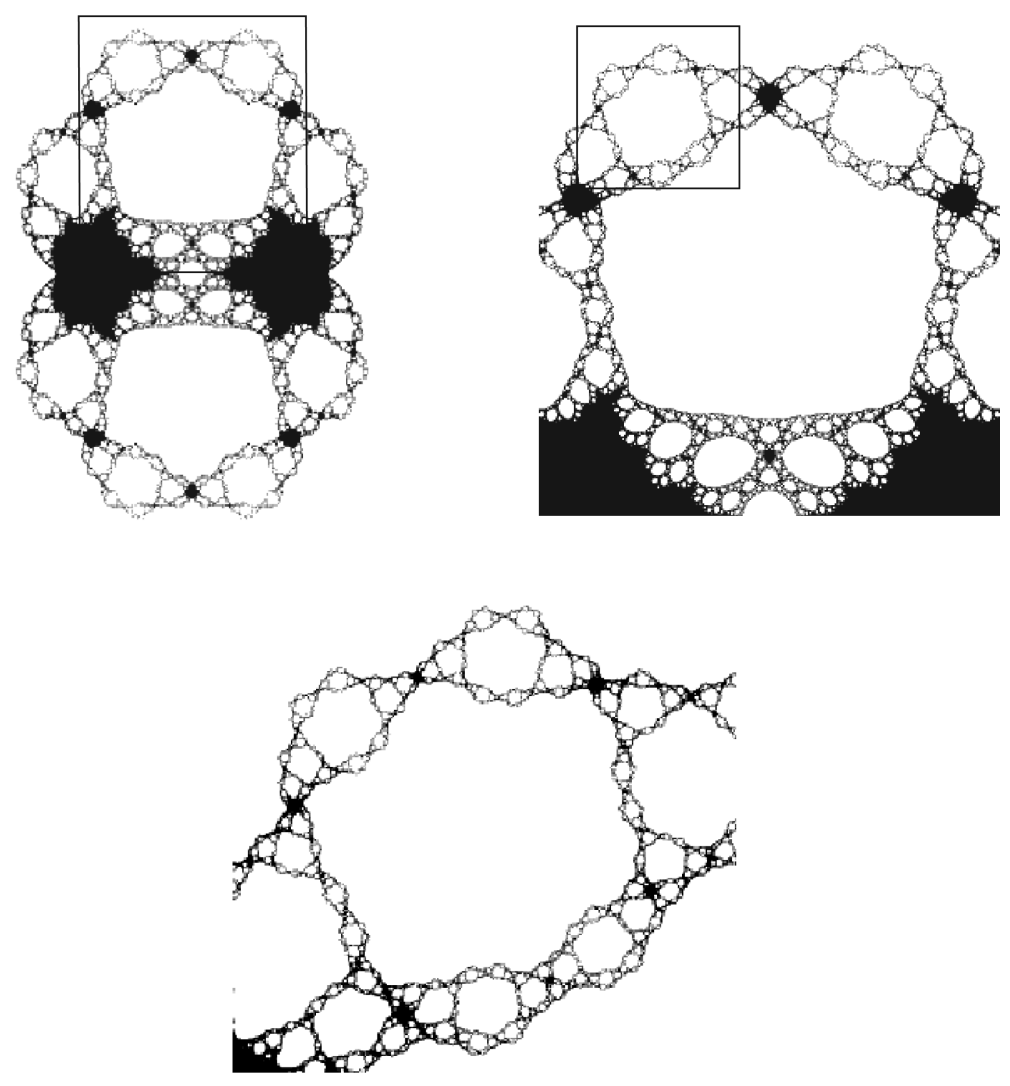

Figure 13. The parameter plane for the family $F_{\lambda}(z)=z^{3}+\lambda / z^{3}$ and a magnification illustrating a Cantor 2 -web together with a further magnification showing a portion of the web.

\section{Invariant CANTOR SETS OF SIMPle ClOSED CURVES}

In Section 4 we showed the existence of a countable collection of special simple closed curves in both the dynamical and parameter planes for the families $F_{\lambda}$ when $n \geq 3$. In this section we show the existence of a very different collection of simple closed curves in these planes. Now we can allow $n \geq 2$. One major difference in this case is that, in both planes, this set of simple closed curves will be a Cantor set of such curves. Moreover, in the dynamical plane, these sets of closed curves will now form an invariant set, and, as we show in the following section, the parameters that lie on the analogous sets of curves in the parameter plane are quite different from those that lie on the Mandelpinski necklaces.

To construct the invariant Cantor set of closed curves in the dynamical plane, recall that we have the critical circle $C_{\lambda}$ that is mapped $2 n$-to-one onto the critical line. If the critical values that serve as the endpoints of the critical line lie inside the critical circle, then we have a countable collection of successive preimages $\zeta_{\lambda}^{k}$ of $C_{\lambda}=\zeta_{\lambda}^{0}$ for $k=1,2,3, \ldots$ These curves each lie outside $C_{\lambda}$, extend outward to the boundary of $B_{\lambda}$ as $k \rightarrow \infty$, and satisfy $F_{\lambda}\left(\zeta_{\lambda}^{k}\right)=\zeta_{\lambda}^{k-1}$. We also have a 
similar set of preimages $\zeta_{\lambda}^{-k}$ extending inward to the boundary of $T_{\lambda}$, where now $F_{\lambda}\left(\zeta_{\lambda}^{-k}\right)=\zeta_{\lambda}^{k-1}$ with $k \geq 1$

To produce the simplest invariant Cantor set of circles in the dynamical plane, assume that the critical values lie inside the curve $\zeta_{\lambda}^{-2}$. Hence $F_{\lambda}\left(v_{\lambda}\right)$ lies outside the closed curve $\zeta_{\lambda}^{1}$. Let $B_{1}$ denote the annulus bounded by $\zeta_{\lambda}^{1}$ and $C_{\lambda}$, and let $B_{0}$ denote the annulus bounded by $\zeta_{\lambda}^{-2}$ and $C_{\lambda}$. Then we have that $F_{\lambda}$ maps $B_{1}-C_{\lambda}$ as an $n$-to-one covering of the disk bounded by $C_{\lambda}$ minus the critical line. Hence there is a preimage of $B_{0}$ lying inside $B_{1}$. Call this preimage $A_{1}$. Since $F_{\lambda}$ is an $n$-to-one covering map on $B_{1}$, it follows that $A_{1}$ is also an annulus. In similar fashion, $F_{\lambda}$ takes $B_{0}-C_{\lambda}$ in $n$-to-one fashion over the disk bounded by $\zeta_{\lambda}^{1}$ minus the critical line. Hence there is another annulus $A_{0} \subset B_{0}$ that is mapped as an $n$-to-one covering of $B_{0} \cup B_{1}$. Continuing in this fashion, there is a subannulus of $A_{1}$ that is mapped as an $n$-to-one covering of $A_{0}$, while there are a pair of subannuli of $A_{0}$, one of which is mapped as an $n$-to-one covering of $A_{0}$, the other to $A_{1}$. Continuing to take preimages, arguments similar to those used to describe the Julia set when $\lambda$ lies in the McMullen domain then say that

$$
\Lambda_{\lambda}^{1}=\bigcap_{j=0}^{\infty} F_{\lambda}^{-j}\left(A_{0} \cup A_{1}\right)
$$

(where $F_{\lambda}^{-1}$ denotes the appropriate preimages in $A_{0}$ or $A_{1}$ ) is an invariant Cantor set of simple closed curves.

The dynamical behavior on $\Lambda_{\lambda}^{1}$ is easy to describe. We can first identify each curve in $\Lambda_{\lambda}^{1}$ by its itinerary. This will now be an infinite sequence $\left(s_{0} s_{1} s_{2} \cdots\right)$ of 0 's and 1's where the digit $s_{j}$ specifies that the $j$ th iterate of this curve lies in either $A_{0}$ or $A_{1}$. Since $A_{1}$ is only mapped over $A_{0}$, in this sequence, if $s_{j}=1$, then $s_{j+1}=0$. But if $s_{j}=0$, then $s_{j+1}$ can be either 0 or 1 . Thus the set of allowable itineraries consists of all sequences of 0 's and 1's where a 1 is never followed by a 1 . Note that the image under $F_{\lambda}$ of the curve with itinerary $\left(s_{0} s_{1} s_{2} \cdots\right)$ is the curve whose itinerary is $\left(s_{1} s_{2} s_{3} \cdots\right)$. The map that takes the itinerary of a curve to the itinerary of its image under $F_{\lambda}$ is what is known as a subshift of finite type on the space of all allowable sequences of 0 's and 1's. Then, on each closed curve, the map is conjugate to either $\theta \mapsto n \theta$ or to $-n \theta$, so we can identify each point on the circle with a natural parameterization of the circle relative to this conjugacy; see [18] for details of this construction.

We can construct in similar fashion larger invariant sets $\Lambda_{\lambda}^{k}$ of simple closed curves as follows. Assume now that the critical value lies inside the curve $\zeta_{\lambda}^{-k-1}$. Let $B_{0}$ be the annulus bounded by $\zeta_{\lambda}^{-k-1}$ and $C_{\lambda}$. Let $B_{1}$ be the annulus bounded by $C_{\lambda}$ and $\zeta_{\lambda}^{1}$ as above. But now, for $j=2, \ldots, k$, let $B_{j}$ be the annulus bounded by $\zeta_{\lambda}^{j-1}$ and $\zeta_{\lambda}^{j}$. Then essentially the same argument as above produces a larger invariant set of simple closed curves $\Lambda_{\lambda}^{k}$ in the dynamical plane. Note that the itineraries of points in $\Lambda_{\lambda}^{k}$ now are given by infinite sequences $\left(s_{0} s_{1} s_{2} \cdots\right)$ where $s_{j}$ is now an integer $0,1, \ldots, k$. In these sequences 0 may be followed by any integer $0,1, \ldots, k$, whereas $j \neq 0$ can only be followed by $j-1$. Clearly, $\Lambda_{\lambda}^{k-1}$ is an invariant subset of $\Lambda_{\lambda}^{k}$.

Now we move again to the parameter plane. In the first case we considered, we have $v_{\lambda}$ lying inside $\zeta_{\lambda}^{-2}$ so that $F_{\lambda}\left(v_{\lambda}\right)$ lies in the exterior of $\zeta_{\lambda}^{1}$. Then there are infinitely many preimages of $\Lambda_{\lambda}$ lying in this external region. Using the coordinates 
described above, we may then find unique $\lambda$-values for which $F_{\lambda}\left(v_{\lambda}\right)$ lands on a particular preimage of a point in $\Lambda_{\lambda}$. This then produces similar collections of curves in the parameter planes for these maps, just as in the previous sections. But, as we shall see in the next section, Julia sets corresponding to parameters on these curves are different from those lying in the Mandelpinski necklaces.

\section{A MYRIAD OF SiERPIŃSKI CURVES}

In Section 4 we saw that the Mandelpinski necklaces in the parameter plane passed through infinitely many Sierpiński holes, i.e., open sets of parameters for which the corresponding Julia sets were all Sierpiński curves. It turns out that Sierpiński curves arise in all of the other types of sets in parameter plane that we have thus far described.

In each Mandelpinski necklace $\mathcal{S}_{k}$ we saw that there were many baby Mandelbrot sets attached to $\mathcal{S}_{k}$. Each such set has a main cardioid in which all parameters have an attracting cycle of some period. Each of the basins of this cycle is then bounded by a simple closed curve. Since the baby Mandelbrot set containing these parameters is "buried" when $k \geq 2$, i.e., this Mandelbrot set does not touch the external boundary of the connectedness locus, it is known that these basin boundaries are pairwise disjoint and, moreover, they cannot touch any of the boundaries of $B_{\lambda}$ and its preimages. Consequently, each parameter drawn from the main cardioid of a Mandelbrot set in these Mandelpinski necklaces has a Julia set that is also a Sierpiński curve. So these Julia sets are homeomorphic to those drawn from any of the Sierpiński holes in the Mandelpinski necklaces. But now the corresponding maps are dynamically very different since there are boundaries of the attracting basin of the cycle that are invariant under some iterate of $F_{\lambda}$. In the case where the parameter was drawn from a Sierpiński hole, the only invariant such boundary was the boundary of $B_{\lambda}$.

As a remark, there are many other buried baby Mandelbrot sets in these parameter planes that do not lie along the Mandelpinski necklaces. Julia sets corresponding to parameters from the main cardioids of these Mandelbrot sets are also Sierpiński curves.

In the case of Cantor webs (or necklaces when $n=2$ ), we find additional parameters whose Julia sets are Sierpiński curves. Obviously, those parameters in the Sierpiński hole portion of the Cantor web have Julia sets that are Sierpiński curves exactly as before. But now consider a parameter that is a buried point in the Cantor set portion of the web. For these parameters, the critical orbit lands on a point in the Cantor set portion of the web in the dynamical plane. Thus the only components of the corresponding Fatou set are the escaping domains, and because the critical points land on buried points, the boundaries of these regions cannot meet. Moreover, the critical orbit cannot be recurrent since the critical points do not lie in the web. So the postcritical set is disjoint from $J\left(F_{\lambda}\right)$. Applying the results in 42 and the other techniques above, we again have a Sierpiński curve Julia set. These, however, are also dynamically quite different from the earlier ones in that they are "structurally unstable". This means that a small change in $\lambda$ causes a significant change in the behavior of the critcal orbit. This orbit now (often) no longer lands on a buried point in the web, but it may land in one of the Sierpiński holes, on a nonburied point, or on some other point that is not in the Cantor web. 
Therefore the topology of and the dynamics on the corresponding Julia set often changes significantly when the parameter moves off a buried point.

Incidentally, if a parameter lies on a nonburied point in the Cantor set portion of the web, then the corresponding Julia set is no longer a Sierpiński curve since certain of the boundaries of the escape regions now touch, so they are not pairwise disjoint. These types of Julia sets are called hybrid Sierpiński curves; see [13.

In Section 6 we described Cantor sets of simple closed curves in the parameter plane. If $\lambda$ lies on such a curve, then again the Julia set of $F_{\lambda}$ is a Sierpiński curve. The reason is that, as above, the critical orbit no longer escapes but rather lands in the Julia set and is nonrecurrent. So $J\left(F_{\lambda}\right)$ is locally connected and the Fatou set consists only of the escaping domains. Since the critical orbits do not lie on the boundary of an escaping domain, the Fatou set is again a collection of disjoint disks whose boundaries are pairwise disjoint, so all of these parameters also correspond to Sierpiński curve Julia sets.

Note, however, that where the critical orbit lands in the invariant Cantor set leads to very different behaviors for the critical orbits. For example, it can be shown that, arbitrarily close to any parameter that lies on one of these curves, there is a parameter for which $v_{\lambda}$ lands on a repelling cycle and another parameter for which $v_{\lambda}$ lands on a point whose orbit is dense in the invariant set of curves. As a consequence, the dynamical behavior on the Sierpinski curve Julia set is very different as soon as $\lambda$ changes, so we again have structural instability. Also, as in the Mandelpinski case, only those parameters that lie on a specific curve and are symmetrically located by either rotation by certain $(n-1)$-st roots of unity or by complex conjugation have topologically conjugate dynamics. Thus we have uncountably many different types of dynamical behaviors in this set of Sierpiński curve parameters.

Thus we see that Sierpiński curve Julia sets arise in a myriad of different ways in the parameter planes for $F_{\lambda}$. To summarize:

Theorem. The Julia set of $F_{\lambda}$ is a Sierpinski curve if:

- $\lambda$ lies in a Sierpiński hole;

- $\lambda$ lies in the main cardioid of a buried baby Mandelbrot set;

- $\lambda$ lies at a buried point in a Cantor necklace or web;

- $\lambda$ lies in the Cantor set of simple closed curves in the parameter plane that corresponds to an $F_{\lambda}$-invariant Cantor set of curves $\Lambda_{\lambda}^{k}$ in the dynamical plane.

All of these Julia sets are the same topologically, but they all have dramatically different dynamical behaviors from nearby parameters (when $\lambda$ does not lie is a specific Sierpiński hole or baby Mandelbrot set).

\section{SiERPIŃSKI GASKET-LIKE JULIA SETS}

There is another well-known fractal object associated with the name Sierpiński, namely the Sierpiński gasket (or triangle) displayed in Figure 14. To construct this set, start with a triangular region in the plane. Subdivide this region into four equal-sized sub-triangles and remove the open middle triangle. This leaves behind three closed and congruent triangles. There are three special points in the remaining set that connect the external and internal complementary regions; these are called the connecting points. Then repeat this process infinitely often, each time 


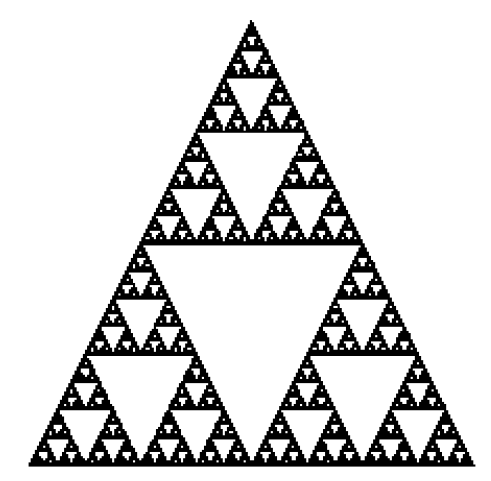

FiguRE 14. The Sierpiński gasket.

removing the open middle triangle from each remaining triangle. The limiting set is the Sierpiński gasket.

A homeomorphic copy of the Sierpiński gasket arises in the family $H_{\lambda}(z)=$ $z^{2}+\lambda / z$. There are three critical points for $H_{\lambda}$ that are given by $(\lambda / 2)^{1 / 3}$. And there are now three distinct critical values given by $3(\lambda / 2)^{2 / 3}$ in contrast with the family $F_{\lambda}$, where there are always only two critical values no matter what the value of $n$ is. However, the Julia set of $H_{\lambda}$ is still symmetric under rotation by a cube root of unity.

When $\lambda \in \mathbb{R}^{-}$, there is a unique critical point $c_{\lambda}$ that lies in $\mathbb{R}^{-}$. The two other critical points, $c_{\lambda}^{ \pm}$, are symmetrically located because of the 3 -fold symmetry. The graph of $H_{\lambda}$ shows that there is a unique parameter $\gamma \approx-0.5925$ for which the critical value $v_{\gamma}=H_{\gamma}\left(c_{\gamma}\right)$ is the unique fixed point lying in $\mathbb{R}^{+}$. See Figure 15, Then one checks easily that the other two critical points both map to a 2-cycle $z_{\gamma}^{ \pm}$, each point of which is a rotation of the fixed point $v_{\gamma}$ by a third of a turn.

The graph of $F_{\gamma}$ also shows that $v_{\gamma}$ lies on the boundary of the basin of $\infty, B_{\gamma}$. Hence the critical point $c_{\gamma}$ lies in the intersection of $\partial T_{\gamma}$ and $\partial B_{\gamma}$. The other two critical points also connect $T_{\gamma}$ and $B_{\gamma}$. Any other point that connects $T_{\gamma}$ to $B_{\gamma}$

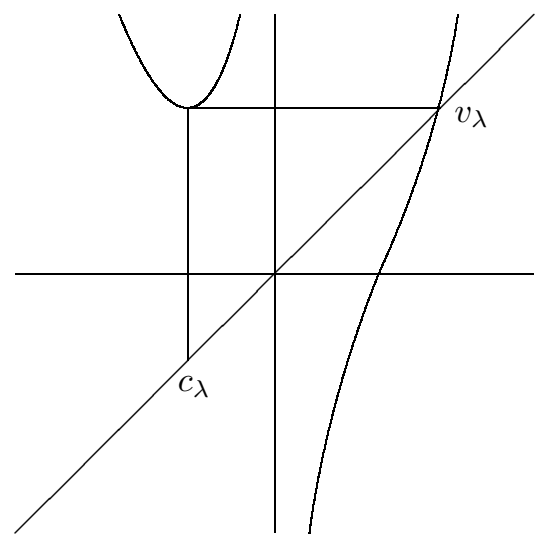

FiguRE 15. The graph of $H_{\gamma}$ when $\gamma=-0.5925$. 


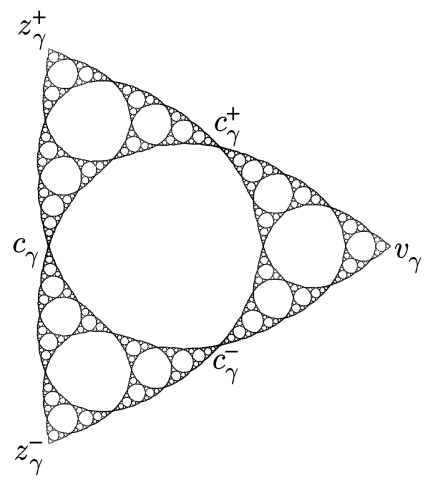

Figure 16. The Julia set for $z^{2}+\gamma / z$ is homeomorphic to the Sierpiński gasket when $\gamma \approx-0.5925$.

would necessarily be a critical point since $F_{\gamma}\left(T_{\gamma}\right)=F_{\gamma}\left(B_{\gamma}\right)=B_{\gamma}$, so the three critical points are the only connecting points between $B_{\gamma}$ and $T_{\gamma}$, just as in the case of the Sierpiński gasket.

Since the critical points all eventually map to periodic points, it follows that the Fatou set consists solely of $B_{\gamma}$ and all of its preimages. We can thus use this fact together with a replica of the construction of the Sierpiński gasket to show that $J\left(H_{\gamma}\right)$ is homeomorphic to the gasket.

As before, it is known that $\partial B_{\gamma}$ is a simple closed curve. So, to construct $J\left(H_{\gamma}\right)$, we begin with the closed disk $\mathbb{C}-B_{\gamma}$. This is our initial "triangle". Then we remove the open triangle $T_{\gamma}$. This leaves three symmetrically located closed sets joined by the connecting points that are the critical points. Then each of these three remaining sets is mapped one-to-one onto $\mathbb{C}-B_{\gamma}$. So, from each of these three sets, we remove the set that is mapped to $T_{\gamma}$; this removes an open set from each of these three sets whose boundary contains exactly three points that connect to either $B_{\gamma}$ or $T_{\gamma}$; two of these points lie in $\partial B_{\gamma}$ and one lies in $\partial T_{\gamma}$ since $H_{\lambda} \mid B_{\lambda}$ is two-to-one while $H_{\lambda} \mid T_{\lambda}$ is one-to-one. Continuing in this manner shows that $J\left(H_{\gamma}\right)$ is homeomorphic to the Sierpiński gasket. See Figure [16.

It turns out that there are many Sierpiński gasket-like sets that arise as Julia sets in the family $F_{\lambda}(z)=z^{n}+\lambda / z^{n}$. By a Sierpinski gasket-like set, we mean a set that is homeomorphic to the following model. Start with the closed unit disk $D_{0}$ in $\mathbb{C}$. Construct a simple closed curve $\nu_{0}$ lying in $D_{0}$ that is symmetric under rotation by a $(2 n)$-th root of unity and that meets $\partial D_{0}$ at exactly $2 n$ connecting points. Remove the interior of this curve from $D_{0}$. This leaves behind $2 n$ symmetrically located closed disks $D_{1}^{j}$ for $j=1, \ldots, 2 n$. Then construct $2 n$ symmetrically located closed curves $\nu_{1}^{j} \subset D_{1}^{j}$, each of which touches the boundary of $D_{1}^{j}$ in exactly $2 n$ points that are different from the original connecting points. Some of these new connecting points will lie in the outer boundary of $D_{1}^{j}$; the remaining new connecting points will lie in the inner boundary. The numbers of these new connecting points that touch each boundary may differ depending on the construction, but these numbers are the same in each $D_{1}^{j}$ due to the symmetry. Then remove the open set in the interior of each $\nu_{1}^{j}$. 
Then continue with this process, each time removing symmetrically located open sets with $2 n$ connecting points touching the boundary of the prior closed set. The limiting set is a Sierpiński gasket-like set. The following result was proved in [26]

Theorem. For $F_{\lambda}(z)=z^{n}+\lambda / z^{n}$, assume that the critical points all lie in $\partial T_{\lambda} \cap \partial B_{\lambda}$ and that each critical point eventually lands on a periodic point. Then the Julia set of $F_{\lambda}$ is homeomorphic to a Sierpinski gasket-like set. Moreover, if two such parameters are not symmetrically located in the parameter plane with respect to rotation by an $(n-1)$-st root of unity or by complex conjugation, then the corresponding Julia sets are not homeomorphic.

Basically, the same construction that we used to construct the Julia set that was homeomorphic to the Sierpiński gasket works in this case. The arrangement of the periodic orbits in $\partial B_{\lambda}$ on which the critical points eventually land dictates how the curves (the preimages of $\partial B_{\lambda}$ ) meet the boundaries of the remaining disks at each stage.

Then, it can be shown that, if two such parameters have critical orbits with a different arrangement relative to the original connecting points (up to symmetry), their Julia sets are not homeomorphic.

See Figure 17 for an example of two Sierpiński gasket-like Julia sets in the case $n=2$ that are not homeomorphic. Note that, in either case, the sets $\mathbb{C}-$ $\left(B_{\lambda} \cup T_{\lambda} \cup F_{\lambda}^{-1}\left(T_{\lambda}\right)\right)$ appear to be homeomorphic. Here $F_{\lambda}^{-1}\left(T_{\lambda}\right)$ consists of the four largest white regions excluding $B_{\lambda}$ and $T_{\lambda}$. Then, how the next preimages of $T_{\lambda}$ are removed changes the topological structure; the connecting points that lie on the various boundaries of $\mathbb{C}-\left(B_{\lambda} \cup T_{\lambda} \cup F_{\lambda}^{-1}\left(T_{\lambda}\right)\right)$ at the next level of the construction are arranged in different ways in these two cases.

The main ingredient in the proof of this theorem is the fact that any homeomorphism between two such Julia sets must map the first set of connecting points to the corresponding set of connecting points. To prove that the corresponding maps are not conjugate on their Julia sets, this step would be obvious since the connecting points are the critical points. But the proof that these sets are not homeomorphic relies on the fact that the connecting points are the only $2 n$ points whose removal
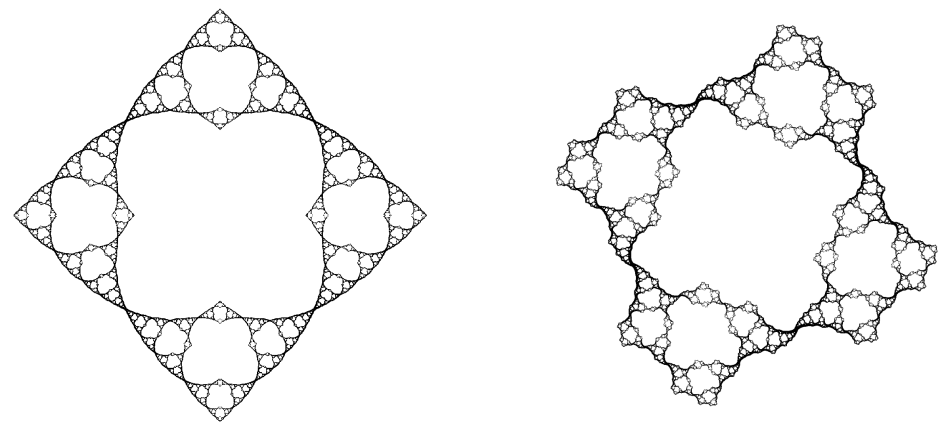

Figure 17. These two Sierpiński gasket-like Julia sets from the family with $n=2$ are not homeomorphic. Here $\lambda \approx-0.3642$ and $\lambda \approx-0.0196+0.2754 i$. In the first case, some of the second preimages of $T_{\lambda}$ have connecting points that lie in $\partial B_{\lambda}, \partial T_{\lambda}$, and $\partial F_{\lambda}^{-1}\left(T_{\lambda}\right)$ while there are no such preimages in the second case. 
from the Julia set separates the remaining set into $2 n$ disjoint sets. Removal of any other collection of $2 n$ points would not accomplish this. Then, at the next level, it is only the first preimages of $T_{\lambda}$ that have $2 n$ connecting points, so these too must be preserved by any homeomorphism. Then this process continues to the limit.

As a remark, since we have assumed that the critical points lie in $\partial T_{\lambda} \cap \partial B_{\lambda}$, it follows that the corresponding parameters lie on the boundary of the connectedness locus in the parameter plane. Numerically, these are the parameters that lie at the tips of the outwardly protruding spokes visible along the boundary of the connectedness locus.

\section{ThE CRAZY CASE $n=2$}

In most of the previous sections, we have considered the family of singularly perturbed maps of the form $z^{n}+\lambda / z^{n}$ where $n \geq 3$. It turns out that the case where $n=2$ is much more complicated. In this section we describe three major differences between the cases $n=2$ and $n>2$. The first is the fact that there is no McMullen domain when $n=2$. As we showed in Section 2, we have $F_{\lambda}\left(v_{\lambda}\right) \rightarrow \infty$ as $\lambda \rightarrow 0$ when $n>2$, but $F_{\lambda}\left(v_{\lambda}\right) \rightarrow 1 / 4$ as $\lambda \rightarrow 0$ when $n=2$. So $v_{\lambda}$ does not lie in $T_{\lambda}$ when $n=2$ as it does when $n>2$, and therefore there is no McMullen domain surrounding the origin. In particular, the large Mandelbrot set in the parameter plane for $n=2$ (see Figure 18) extends all the way to the origin (so technically this is not a complete Mandelbrot set since the "tip of the tail" of this set lands at $\lambda=0)$.

Another major difference involves the Mandelpinski necklaces described in Section 4. Yes, there do exist Mandelpinski necklaces when $n=2$ (surrounding the origin in this case, not a McMullen domain); see [11. But the configuration of these necklaces is very different when $n=2$. Recall that we showed that the $k$ th Mandelpinski necklace passes through exactly $(n-2) n^{k-1}+1$ centers of Sierpiński
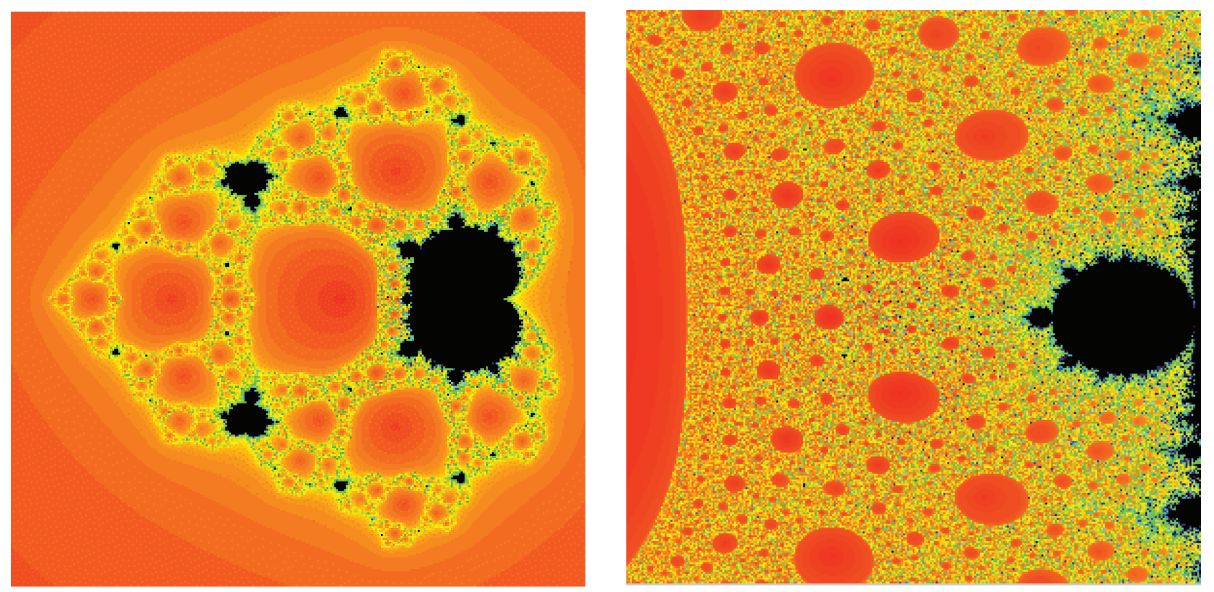

FiguRE 18. The parameter plane and a magnification around the origin for the family $z^{2}+\lambda / z^{2}$. The large central disk on the left is a Sierpiński hole, not a McMullen domain. 
holes and baby Mandelbrot sets. So, when $n=2$, this means that each Mandelpinski necklace passes through just one center of a Sierpiński hole and one baby Mandelbrot set for each $k$. Not nearly as interesting!

Note that this does say that, in any neighborhood of the origin in the parameter plane when $n=2$, there are infinitely many dynamically distinct Sierpiński holes and baby Mandelbrot sets. So this case truly does yield a singular perturbation since, when $\lambda \neq 0$, we find an incredible variety of different types of Julia sets; the corresponding dynamical behavior is dramatically different as we move in different directions away from 0. Moreover, the proof we gave earlier of the existence of Cantor sets of simple closed curves in the parameter plane on which each parameter corresponds to a map that has a Sierpiński curve Julia set also holds in any neighborhood of the origin when $n=2$. So the dynamical behavior truly explodes when $\lambda$ becomes nonzero. See Figure 18 for a magnification of the parameter plane about 0 when $n=2$.

Perhaps the biggest difference between the cases $n=2$ and $n>2$ occurs in the dynamical plane. The following result is proved in [21].

Theorem. (1) In the case $n=2$, if $\lambda_{j}$ is a sequence of parameters converging to 0 , then the Julia sets of $F_{\lambda_{j}}$ converge as sets (in the Hausdorff topology) to the closed unit disk.

(2) However, if $n>2$, this is not the case. Specifically, for a given punctured neighborhood $U$ of 0 in the McMullen domain, there exists $\delta>0$ such that, for each $\lambda \in U$, there is a round annulus (i.e., an annulus bounded by actual circles) in the complement of the Julia set inside the unit circle whose internal and external radii differ by at least $\delta$.

So when $n=2$ the Julia sets of $F_{\lambda}$ get closer and closer to the closed unit disk as $\lambda \rightarrow 0$, but, of course, when $\lambda=0$, the Julia set is just the unit circle. This is somewhat surprising since it is well known that, should a Julia set ever contain an open set, no matter how small, then the Julia set must be the entire complex plane. This follows from Montel's Theorem. When $n=2$ we find Julia sets getting arbitrarily close to the entire unit disk as $\lambda \rightarrow 0$, but, of course, never equaling the unit disk. Figure 1 displays two Julia sets in this family where $|\lambda|$ is small.

The proof of convergence to the unit disk is fairly straightforward. Suppose the Julia sets of $F_{\lambda}$ do not converge to the unit disk as $\lambda \rightarrow 0$. Then there exists $\delta>0$ and a sequence of parameters $\lambda_{j} \rightarrow 0$ such that the Fatou set for each $F_{\lambda_{j}}$ contains a disk about some point $z_{j}$ lying on or inside the unit circle whose radius is at least $\delta$. Call this disk $B_{\delta}\left(z_{j}\right)$. Then, since the unit disk is compact, there is a subsequence of the $z_{j}$ that converges to some point $z^{*}$ in the unit disk. One checks easily that $z^{*} \neq 0$ since $T_{\lambda}$ becomes arbitrarily small as $\lambda \rightarrow 0$.

Thus we may assume at the outset that there is a sequence of parameters $\lambda_{j}$ such that the Fatou set of $F_{\lambda_{j}}$ contains a disk of radius $\delta>0$ about $z^{*}, B_{\delta}\left(z^{*}\right)$. But now, when $\left|\lambda_{j}\right|$ is close to 0 , the map $F_{\lambda_{j}}$ is very close to $z^{2}$ except for points that are very close to the origin. The map $z \mapsto z^{2}$ does move the disk $B_{\delta}\left(z^{*}\right)$ closer to the origin, but it also expands the arguments of points in this disk by a factor of 2 . So, assuming $j$ is sufficiently large, $F_{\lambda_{j}}$ eventually maps $B_{\delta}\left(z^{*}\right)$ to an annulus that surrounds the origin. So this annulus lies in the Fatou set. This would then disconnect the Julia set, but it is known [28] that these Julia sets are always connected sets. This contradiction gives the result. 
For the case $n>2$ we have seen that the Fatou set contains a countable number of annuli (as well as the disks $B_{\lambda}$ and $T_{\lambda}$ ) when $\lambda$ is small. These annuli are mapped one to another as an $n$-to-one covering until they eventually map to $T_{\lambda}$. Then, using the concept of the modulus of an annulus and a result of Blé and Douady [9], one can show that, for $|\lambda|$ small, there is always an annulus in the Fatou set that is located outside a given disk about the origin whose modulus is bounded away from 0 . This provides a "large" open set in the Fatou set for all such parameters.

In the more general family $z^{n}+\lambda / z^{d}$ where $n \geq 2, d \geq 1$, there is always a McMullen domain when $n, d \geq 2$ but $n$ and $d$ are not both equal to 2 . When $d=1$, however, there is again no McMullen domain. An easy computation shows that $F_{\lambda}^{2}\left(c_{\lambda}\right) \rightarrow 0$ unlike the McMullen domain case where $F_{\lambda}^{2}\left(c_{\lambda}\right) \rightarrow \infty$ as $\lambda \rightarrow 0$. But the dynamical behavior near $\lambda=0$ is very different. It is known [25] that the Julia sets converge to the closed unit disk only when $\lambda \rightarrow 0$ along the $(n-1)$ rays given by $t \nu$ where $\nu$ is an $(n-1)$-st root of unity. Along all other rays in the parameter plane, there are always large attracting basins extending from $T_{\lambda}$ to $B_{\lambda}$, so $J\left(F_{\lambda}\right)$ does not converge to the unit disk along these rays.

\section{More General Singular Perturbations}

In this section we consider the more general family

$$
G_{\lambda, c}(z)=z^{n}+c+\frac{\lambda}{z^{n}} .
$$

Here the most interesting behavior occurs when $c$ is chosen at the center of a hyperbolic component of the Multibrot set, i.e., where $c$ is a parameter for which the critical point of $z^{n}+c$ lies on a periodic orbit (a superattracting cycle).

When $c$ lies in a hyperbolic component but not at its center, the dynamical behavior of $G_{\lambda, c}$ is much simpler. As an example of this, consider the special case where $c \neq 0$ lies in the main cardioid of the Multibrot set. So the map $z^{n}+c$ has an attracting fixed point $z_{c} \neq 0$. In this case, the orbit of 0 now tends to this fixed point and the Julia set is a simple closed curve surrounding the basin of attraction of $z_{c}$. For $\lambda \neq 0$ and small, $G_{\lambda, c}$ still has an attracting fixed point $z_{\lambda, c}$ (unlike the case of $G_{\lambda, 0}$ where this fixed point has become a pole). Thus some of the newly born critical points of $G_{\lambda, c}$ must tend to this fixed point. In fact, it can be shown that, for $\lambda$ small enough, all such critical orbits do so. Meanwhile, since $G_{\lambda, c}(z) \approx z^{n}+c$ when $\lambda$ is small and $z$ is not close to 0 , there still is a simple closed curve in $J\left(G_{\lambda, c}\right)$ that forms the boundary of the immediate basin of $\infty$. But, since the degree of the map has risen, there are other preimages of this simple closed curve in $J\left(G_{\lambda, c}\right)$. In fact, it can be shown that the Julia set now consists of countably many other disjoint copies of this curve (only one of which surrounds the origin) together with an uncountable set of point components that accumulate everywhere on these curves (see [43]). So this type of Julia set is very different from (and much simpler than) the Julia sets that arise when the pole is placed at a point on the superattracting cycle.

There are several things that make the family $G_{\lambda, c}$ more complicated than the previously discussed maps $F_{\lambda}$ where $c=0$. The first is that there are now two distinct free critical orbits instead of just one. The critical points are given, as before, by $\lambda^{1 / 2 n}$, but now the critical values are $\pm v_{\lambda}=c \pm 2 \sqrt{\lambda}$. Since $c \neq 0$, the orbits of $\pm v_{\lambda}$ no longer behave symmetrically. It can happen, for example, that one of these critical orbits lies in the immediate basin of $\infty$ while the other lies in the 

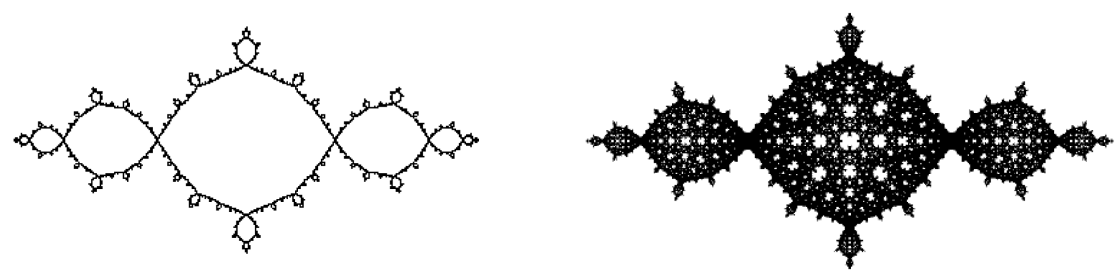

Figure 19. The Julia sets for $z^{2}-1$ and for $z^{2}-1-.001 / z^{2}$.

basin of an attracting cycle. This produces an infinitely connected Julia set that is neither a Cantor set nor a Cantor set of circles, something that never occurs for the maps $F_{\lambda}$.

A second difference involves the boundary of the immediate basin of $\infty$ which we now denote by $\partial B_{\lambda, c}$. When $c=0$, we have seen that this boundary is a simple closed curve (except when the Julia set is a Cantor set). But for other centers of hyperbolic components, this boundary is quite different. When $\lambda=0$, the boundary of the basin at $\infty$ is just the Julia set of $z^{n}+c$ which is never a simple closed curve when $c$ lies outside the main cardioid of the Multibrot set. For $\lambda$ close to 0, the map $G_{\lambda, c}$ is then close to $z^{n}+c$ near the boundary of the basin at $\infty$, so a quasiconformal surgery argument [4] shows that $\partial B_{\lambda, c}$ is now homeomorphic to the Julia set of the unperturbed map $z^{n}+c$. For example, in Figure 19, we display the Julia sets of $z^{2}-1+\lambda / z^{2}$ for $\lambda=0$ and $\lambda=-0.001$. When $\lambda=0$, the critical point 0 lies on a superattracting cycle of period two. The Julia set in this case is known as the basilica. For $\lambda$ small, as always, the Julia set explodes, but the boundary of the basin at $\infty$ is homeomorphic to the basilica; see Figure 19 .

The complement of the basin of $\infty$ for $G_{\lambda, 0}$ (i.e., the Julia set together with all the bounded complementary domains) is known as the filled Julia set. When $c$ lies at the center of a hyperbolic component of period two or higher, the portion of the Fatou set in the filled Julia set contains infinitely many disjoint open disks (the basin of the cycle and all of its preimages).

As in the case of the family $F_{\lambda}$, there is a major difference between the cases $n=2$ and $n>2$ in the family $G_{\lambda, c}$. We have a similar convergence theorem when $n=2($ see $[22])$ :
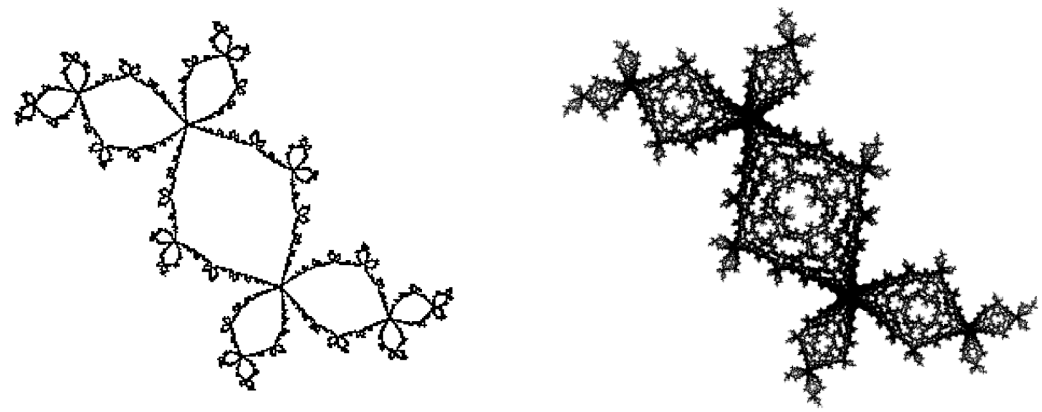

Figure 20. The Julia sets for the $z^{2}-0.12+0.75 i$ (the Douady rabbit) and its singular perturbation. 
Theorem. Suppose $n=2$. Then the Julia set of $G_{\lambda, c}$ converges in the Hausdorff topology to the filled Julia set of $z^{2}+c$ as $\lambda \rightarrow 0$.

For example when $c=-1$ and $\lambda=-0.001$, we see in Figure 19 that the Julia set for $G_{\lambda,-1}$ comes very close to the entire filled Julia set of $z^{2}-1$. Figure 20 shows a similar phenomenon for the quadratic Julia set known as the Douady rabbit.

Note that the fact that $\partial B_{\lambda, c}$ is no longer a simple closed curve affects the structure of the preimages of this set. When $\lambda$ is small, we still have a trap door that is disjoint from $B_{\lambda, c}$, but the boundary of this set is no longer a simple closed curve. Rather, it is an "inverted" copy of the boundary of the basin at $\infty$. Figures 21 and 22 display magnifications of the trap doors for the perturbed basilica and Douady rabbit.

Proving that the Julia sets converge to the filled Julia set of $z^{2}+c$ takes more work than in the case where $c=0$. Here is one issue. Suppose $c$ has the property that $z^{2}+c$ has a superattracting cycle of period $k>1$. Then for $\lambda$ small, there is a trap door surrounding the origin in which all points are mapped by $G_{\lambda, c}$ to
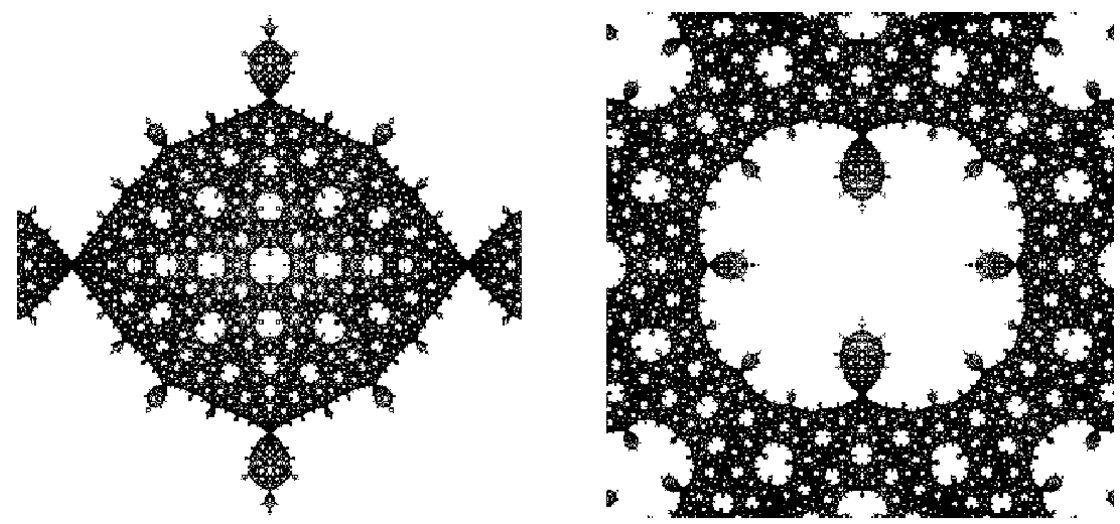

Figure 21. Two magnifications of the trap door for the perturbed basilica.

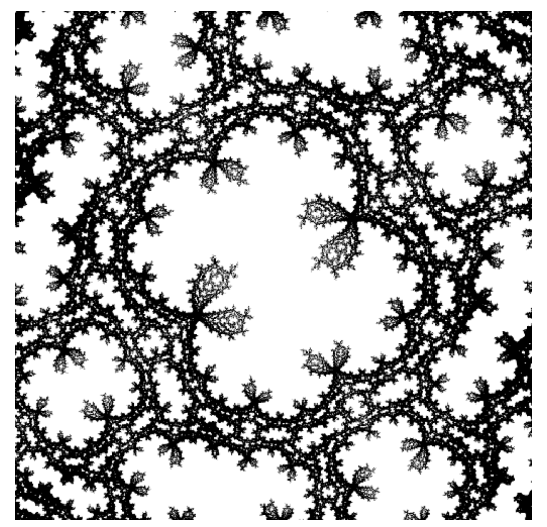

FiguRE 22. A magnifications of the trap door for the perturbed Douady rabbit. 
$B_{\lambda, c}$. When $c=0$, we know that the critical values do not lie in the trap door since $F_{\lambda}\left(v_{\lambda}\right)=1 / 4+4 \lambda$. So the second iterates of the critical points lie well inside the simple closed curve bounding $B_{\lambda}$ when $\lambda$ is small. But what about the orbits of $\pm v_{\lambda}$ when $c \neq 0$ ? Can we have $G_{\lambda, c}^{k}\left(c_{\lambda}\right)$ lying in the trap door? The answer here is no, but the proof involves more complex analysis and is much more complicated.

For a sketch of the proof, assume that $c$ lies on an attracting cycle of period $k>1$ for $G_{0, c}$. A holomorphic motions argument then shows that, for small enough $\lambda$, the set $\partial B_{\lambda, c}$ varies analytically with $\lambda$. When $\lambda=0$, we have $k$ simply connected and disjoint components of the Fatou set that surround the points $G_{\lambda, 0}^{j}(0)$ for $j=0,1, \ldots, k-1$. Denote these sets by $D_{0}^{j}$. We have similar open sets $D_{\lambda}^{j}$ for $G_{\lambda, c}$, but these sets no longer lie in the Fatou set since the dynamical behavior has exploded when $\lambda \neq 0$. However, they are similar sets in that they are each bounded by simple closed curves that lie in $\partial B_{\lambda, c}$ and their boundaries vary analytically with the corresponding boundaries of $D_{0}^{j}$.

In analogy with the situation for $F_{\lambda}$, we need to show that the $(k+1)$-st iterates of the critical points lie in $D_{\lambda}^{1}$ and hence not in $B_{\lambda, c}$. Clearly, for $0 \leq j \leq k$, the $j$ th iterate of the critical points lies in $D_{\lambda}^{j}$. This follows since the first iterate is given by $c \pm 2 \sqrt{\lambda}$, which is close to $c$ when $\lambda$ is close to 0 (and hence inside $D_{\lambda}^{1}$ ). Then the next $j$ iterates of these points are far from the pole and hence their locations can be approximated using $G_{\lambda, 0}^{j}$. So the $j$ th iterate of the critical values are given approximately by

$$
G_{\lambda, c}^{j}(c \pm 2 \sqrt{\lambda}) \approx G_{\lambda, 0}^{j}(c)+2^{j}( \pm 2 \sqrt{\lambda}) \prod_{i=1}^{j} G_{\lambda, 0}^{i}(0)
$$

for $j=1, \ldots, k-1$. In particular, the $(k-1)$-st iterates of the critical values (or the $k$ th iterates of the critical points) are given approximately by

$$
G_{\lambda, c}^{k-1}(c \pm 2 \sqrt{\lambda}) \approx 2^{k-1}( \pm 2 \sqrt{\lambda}) \prod_{i=1}^{k-1} G_{\lambda, 0}^{i}(0)
$$

since $G_{\lambda, 0}^{k-1}(c)=0$. So the critical orbits return very close to the origin at the $k$ th iterate (just as in the case where $c=0$ where the first iterate of the critical points is $\pm 2 \sqrt{\lambda})$.

The claim is that the next iterate lies within the disk $D_{\lambda}^{1}$. Proving this involves first using the Riemann Mapping Theorem and then the Koebe-1/4 Theorem to get an estimate of the size of of $D_{0}^{1}$. This estimate then shows that the $k$ th iterate of the critical values do indeed lie inside $D_{\lambda}^{1}$ and hence, when $n=2$, we again do not have the analogue of the McMullen domain [22. For example, if $c=-1$, then applying $G_{\lambda, c}$ one more time in the above formula shows that $G_{\lambda,-1}^{2}(c \pm 2 \sqrt{\lambda}) \rightarrow-15 / 16$ as $\lambda \rightarrow 0$. The point $-15 / 16$ lies well within the region $D_{\lambda}^{1}$ as it is easily seen that, when $\lambda=0$, this region contains the interval $(-5 / 4,-3 / 4)$ along the negative real axis.

One other major difference between the case $k=1$ and $k>1$ would seem to be that, because $\partial B_{\lambda, c}$ is no longer a simple closed curve, Sierpiński curves no longer arise as Julia set for these maps. While this is true, it turns out that there are many Sierpiński curve subsets of these Julia sets. For example, if both critical orbits eventually escape to $\infty$, then the only Fatou domains are the immediate basin of $\infty$ and all of its preimages. These preimages always have infinitely many attachments. 
However, if we discard all of these attached portions of these preimages, then the leftover components of the Julia set are bounded by simple closed curves and again, this subset of the Julia set is a Sierpiński curve; see 4 for details.

When $n>2$ and $\lambda$ is small, we have a structure in the dynamical plane that is somewhat similar to the Cantor set of simple closed curves that appear when $\lambda$ lies in the McMullen domain when $c=0$. But there are several differences.

The first difference involves the Cantor set of simple closed curves. If $G_{\lambda, 0}$ has an attracting cycle of period $k$, then we can consider the set of points whose orbits under $G_{\lambda, c}$ travel through the disks $D_{\lambda}^{j}$ exactly as does the orbit of 0 under $G_{\lambda, 0}$. Then essentially the same argument given by McMullen [45] when $c=0$ shows that this set of points is a Cantor set of simple closed curves. However, countably many of these curves eventually map to $\partial D_{\lambda}^{0}$. These are the "unburied" curves in this Cantor set that bound the removed annuli and $T_{\lambda}$. Now $\partial D_{\lambda}^{0}$ has infinitely many attachments lying in $\partial B_{\lambda, c}$, so these curves also have infinitely many attachments lying in $J\left(G_{\lambda, c}\right)$. These attachments protrude into $T_{\lambda}$ and the above removed annuli. Thus there are now countably many "decorated" curves in this Cantor set of curves; see Figure 23 .

One can then consider preimages of this Cantor set of decorated curves that lie in all of the other preimages of the disks $D_{\lambda}^{j}$, and this gives an expanded subset of the Julia set. However, these preimages do not produce the entire Julia set. The reason for this is that none of these preimages (except those lying in $\partial B_{\lambda, c}$ ) contain any periodic points; all of these preimages eventually map to the collection of decorated curves lying in $D_{\lambda}^{j}$ for $j=0,1, \ldots, k-1$, and these points have orbits that either remain in the $D_{\lambda}^{j}$ or else map to $\partial B_{\lambda, c}$. So there must be more to the Julia set than just these decorated curves.

The difference here is that there is an uncountable collection of additional point components in the Julia set whose orbits wander around in the interior of the preimages of the $D_{\lambda}^{j}$ in some fashion dictated by how $G_{\lambda, c}$ maps the boundaries of
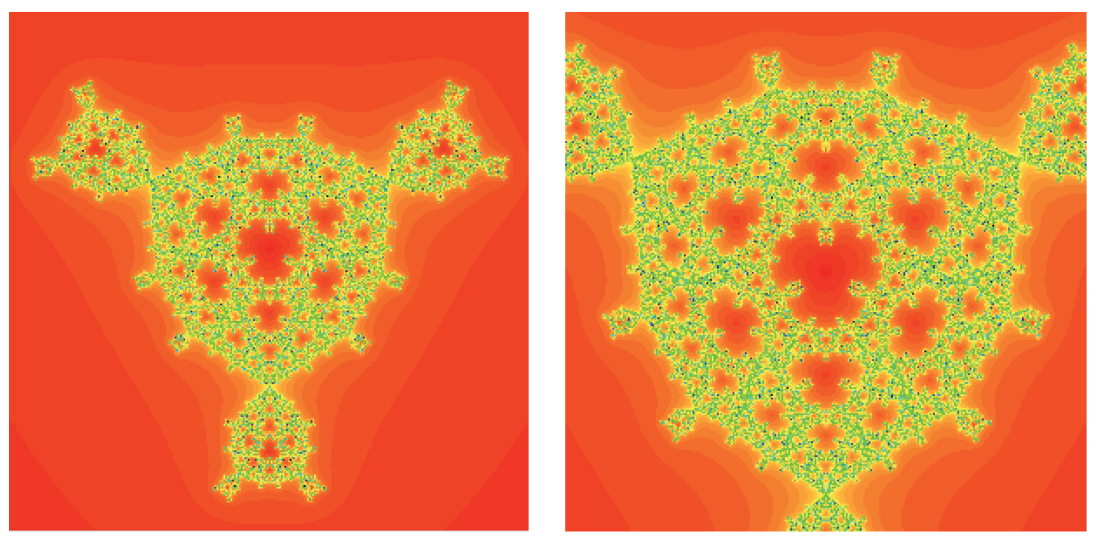

Figure 23. The Julia set for a singular perturbation of $z^{3}-i$ and a magnification. Note the attachments protruding into each annulular region in the Fatou set as well as the trap door and the basin of $\infty$. 
the $D_{\lambda}^{j}$ to other such boundaries. So the Julia sets for these maps contain more than just Cantor sets of decorated curves; see [6].

\section{Open PROBlems}

In this final section, we describe some of the open problems/conjectures regarding singular perturbations. In this paper we have only considered singular perturbations of polynomials. But singular perturbations can occur in all types of maps. One natural question that comes up involves the exponential function $C e^{z}$. These maps hold a similar position in the set of entire functions as the quadratic family $z^{2}+$ $c$ does in the set of polynomials in that there is only one critical orbit. In the exponential case, there are no critical points. However, 0 is now an asymptotic value (the only such point), and its orbit therefore plays a similar role as does the orbit of 0 for $z^{2}+c$. For example, it is well known that, if the orbit of 0 tends to $\infty$ for $C e^{z}$, then the Julia set is the entire complex plane, whereas if the orbit of 0 tends to an attracting cycle, the Julia set is a (pinched) Cantor bouquet. So a natural question would be what happens if we add a pole into this family, say at 0 ? Presumably, one would get a combination of entire and rational dynamics in certain cases. Such an interesting mixture of entire and polynomial dynamics has been seen in some families recently 34. In addition, some of the features we observed earlier, such as Sierpiński curve Julia sets, do arise in other families, such as the meromorphic maps known as the Weierstrass elliptic $p$-functions 38 .

Problem 1. Describe the topology of and the dynamics on the Julia sets for the family of maps $C e^{z}+\lambda / z^{d}$.

One of the major open problems in polynomial dynamics involves the local connectivity of the Mandelbrot set. If the Mandelbrot set could be shown to be locally connected, then we would basically understand everything about quadratic dynamics. In the families of maps $F_{\lambda}$ there are infinitely many small copies of the Mandelbrot set, so we do not yet have a complete grasp of what is happening when parameters lie in these sets. On the other hand, the parameter planes for these maps include much more structure. As we have seen, there are Cantor necklaces and Mandelpinski necklaces throughout this set. And we have a good grasp on the structure of these subsets of the parameter plane. So another natural question would be to combine these two topological objects to produce an entire "map" of the parameter plane. Some progress has been made on this front with the construction of Cantor-Mandelbrot-Sierpiński trees in the parameter planes [20], but there is a lot more work to do.

Problem 2. For the family of maps $z^{n}+\lambda / z^{d}$, describe the arrangement of the Sierpiński holes and Mandelbrot sets in the parameter plane.

Problem 3. As described in Section 3, we have an exact count of the number of Sierpiński holes in the parameter plane for $z^{n}+\lambda / z^{d}$ with escape time $\kappa$. But, as yet, there is no such count for the number of baby Mandelbrot sets in the parameter plane with base period $k$.

There is a major difference in the Julia sets drawn from the main cardioids of the Mandelbrot sets that are buried and those that are "exposed". Here exposed means that the boundary of this Mandelbrot set meets the outer boundary of the connectedness locus. As shown in Section 7, when the baby Mandelbrot set is 
buried, the Julia sets corresponding to parameters in the main cardioid are always Sierpiński curves. But in the exposed case, these Julia sets are "checkerboard" Julia sets. The topological structure of these kinds of Julia sets is very different from the Sierpiński curve Julia sets in that the boundaries of the periodic attracting basins and their preimages now meet infinitely many boundaries of the escaping regions but none of the other boundaries of the attracting basins. Similarly, each boundary of an escaping region touches infinitely many boundaries of the attracting cycle and its preimages but none of the other boundaries of the escaping regions. A natural question would be to give a dynamical and topological clasification of these sets. Some progress has recently been made along these lines in the case of $z^{n}+\lambda / z^{d}$ 3]. In this paper it was shown that the checkerboard Julia sets drawn from the main cardioids of the $n+d$ principal Mandelbrot sets are all homeomorphic, but only symmetrically located cardioids contain parameters with conjugate dynamics. In contrast, some recent work on the family $z^{2}+\lambda / z^{2} 33$ indicates that the Julia sets drawn from the exposed Mandelbrot sets with base period greater than one are not homeomorphic (except in the case of complex conjugate cardioids).

Problem 4. Classify the topology of Julia sets that arise from the main cardioids of exposed Mandelbrot sets.

One of the major tools used to understand the dynamics of quadratic polynomials is provided by the existence of external rays in both the dynamical and parameter planes. These arise as follows. Since there is always a basin of $\infty$ for $z^{2}+c$, by the Riemann Mapping Theorem, there is an analytic homeomorphism that takes the exterior of the unit disk to the immediate basin of $\infty$ when the Julia set is a connected set. As shown in [31, there is a natural extension of this construction to the exterior of the Mandelbrot set. Infinitely many of these external rays are known to limit on a single point in the dynamical and parameter planes. (If the Mandelbrot set were to be locally connected, then all of these rays would land on distinct points.) The landing rays then provide a lot of information about the corresponding geometry of the Julia and Mandelbrot sets. For the rational maps considered in this paper, we have a similar collection of external rays, and as shown in [53], all of these external rays land for the family $z^{n}+\lambda / z^{n}$ when $n \geq 3$. In particular, it follows that the boundary of the connectedness locus in parameter plane is then a simple closed curve. However, in this case, as shown in Section 5, certain of these rays extend into the Julia set all the way down to the origin. Unlike external rays, these internal rays are known to cross each other at infinitely many points. How these internal rays are intertwined should help provide a good map of the internal structure of both the Julia sets and the parameter planes for these maps. For example, these internal rays were used to show 52 that the boundary of $B_{\lambda}$ is always a simple closed curve (provided $\lambda$ does not lie in the Cantor set locus).

Problem 5. Determine the configuration of the internal rays in the dynamical and parameter planes for $z^{n}+\lambda / z^{n}$. In particular, how do these rays make the transit from $\partial B_{\lambda}$ to $\partial T_{\lambda}$ ?

In the parameter planes when $n>2$, the region around the McMullen domain (specifically the region inside the first Mandelpinski necklace) has a very interesting structure. It can be shown that there is more to this story, however. For any Sierpiński hole attached to the necklace $\mathcal{S}^{k}$ with $k>1$, there are infinitely many 
sub-Mandelpinski necklaces surrounding this region. A natural question is whether or not this process continues: is each Sierpiński hole along this subnecklace also surrounded by infinitely many sub-subnecklaces? And so forth.

Problem 6. Determine the structure of the sub-Mandelpinski necklaces in the annular regions bounded by the Mandelpinski necklaces.

Another open question involves the structure of the parameter plane outside the Mandelpinski necklace $\mathcal{S}^{1}$. A glance at the parameter planes in Figure 4 indicates that each of these outer Sierpiński holes are now surrounded by exactly $2 n$ Sierpiński holes with the same escape time. Then these holes are similarly each surrounded by $2 n$ smaller Sierpiński holes. So the external region in the parameter plane appears to be quite different. What is the geometry in this region? And how does this geometry change as we move from outside to inside $\mathcal{S}^{1}$ ?

Problem 7. Determine the structure of the Sierpiński holes and Mandelbrot sets in the region that lies outside the Mandelpinski necklace $\mathcal{S}^{1}$. Are there analogues of Mandelpinski necklaces in these regions?

In Section 7 we showed that there are several different ways that Sierpiński curve Julia sets arise in these families. A natural question is, Are there other ways that these types of sets appear in these families? A related problem involves how the different dynamical behaviors on Sierpiński curve Julia sets can be characterized. As mentioned in Section 3 [50, this is known when the Julia set is an escape time Sierpiński curve. But what characterizes the dynamical difference for other Sierpiński curve Julia sets?

Problem 8. Find a dynamical invariant that differentiates the various types of Sierpiński curve Julia sets.

As a final comment, this area of research is quite accessible to undergraduates. Indeed many of the papers mentioned above were coauthored by undergraduate students, including [5], 22, 25], 33], and [56].

\section{ABOut THE AUTHOR}

Robert L. Devaney received his Ph.D. from University of California, Berkeley, under the direction of Stephen Smale. He is the Feld Family Professor of Mathematics at Boston University and currently serves as president of the Mathematical Association of America.

\section{REFERENCES}

[1] Daniel S. Alexander, Felice Iavernaro, and Alessandro Rosa, Early days in complex dynamics, History of Mathematics, vol. 38, American Mathematical Society, Providence, RI, 2012. A history of complex dynamics in one variable during 1906-1942. MR2857586 (2012k:37103)

[2] Paul Blanchard, Complex analytic dynamics on the Riemann sphere, Bull. Amer. Math. Soc. (N.S.) 11 (1984), no. 1, 85-141, DOI 10.1090/S0273-0979-1984-15240-6. MR741725 (85h:58001)

[3] Blanchard, P., Çilingir, F., Cuzzocreo, D., Devaney, R. L., Look, D. M., and Russell, E. D. Checkerboard Julia Sets for Rational Maps. To appear.

[4] Paul Blanchard, Robert L. Devaney, Antonio Garijo, Sebastian M. Marotta, and Elizabeth D. Russell, The rabbit and other Julia sets wrapped in Sierpiński carpets, Complex dynamics, A K Peters, Wellesley, MA, 2009, pp. 277-295, DOI 10.1201/b10617-10. MR2508261 (2010m:37072) 
[5] Paul Blanchard, Robert L. Devaney, Daniel M. Look, Pradipta Seal, and Yakov Shapiro, Sierpiński-curve Julia sets and singular perturbations of complex polynomials, Ergodic Theory Dynam. Systems 25 (2005), no. 4, 1047-1055, DOI 10.1017/S0143385704000380. MR2158396(2006d:37087)

[6] Paul Blanchard, Robert L. Devaney, Antonio Garijo, and Elizabeth D. Russell, A generalized version of the McMullen domain, Internat. J. Bifur. Chaos Appl. Sci. Engrg. 18 (2008), no. 8, 2309-2318, DOI 10.1142/S0218127408021725. MR2463865(2009k:37101)

[7] Branner, B. and Fagella, N. The Theory of Polynomial-Like Mappings — The Importance of Quadratic Polynomials. In Proc. 7th EWM Meeting, 57-70.

[8] Xavier Buff and Arnaud Chéritat, The Brjuno function continuously estimates the size of quadratic Siegel disks, Ann. of Math. (2) 164 (2006), no. 1, 265-312, DOI 10.4007/annals.2006.164.265. MR2233849 (2008f:37098)

[9] Gamaliel Blé, Adrien Douady, and Christian Henriksen, Round annuli, In the tradition of Ahlfors and Bers, III, Contemp. Math., vol. 355, Amer. Math. Soc., Providence, RI, 2004, pp. 71-76, DOI 10.1090/conm/355/06445. MR2145056 (2006b:31004)

[10] Figen Çilingir, Robert L. Devaney, and Elizabeth D. Russell, Extending external rays throughout the Julia sets of rational maps, J. Fixed Point Theory Appl. 7 (2010), no. 1, 223-240, DOI 10.1007/s11784-010-0003-2. MR2652518(2012g:37091)

[11] Cuzzocreo, D. and Devaney, R. L. Simple Mandelpinski Necklaces for $z^{2}+\lambda / z^{2}$. To appear.

[12] Laura DeMarco, Iteration at the boundary of the space of rational maps, Duke Math. J. 130 (2005), no. 1, 169-197, DOI 10.1215/S0012-7094-05-13015-0. MR2176550 (2006k:37130)

[13] Robert L. Devaney, A myriad of Sierpiński curve Julia sets, Difference equations, special functions and orthogonal polynomials, World Sci. Publ., Hackensack, NJ, 2007, pp. 131-148, DOI 10.1142/9789812770752_0011. MR2451164 (2009j:30058)

[14] Robert L. Devaney, The McMullen domain: satellite Mandelbrot sets and Sierpiński holes, Conform. Geom. Dyn. 11 (2007), 164-190 (electronic), DOI 10.1090/S1088-4173-07-00166-X. MR2346215 (2008f:37106)

[15] Robert L. Devaney, Baby Mandelbrot sets adorned with halos in families of rational maps, Complex dynamics, Contemp. Math., vol. 396, Amer. Math. Soc., Providence, RI, 2006, pp. 37-50, DOI 10.1090/conm/396/07392. MR2209085 (2006k:37131)

[16] Robert L. Devaney, Structure of the McMullen domain in the parameter planes for rational maps, Fund. Math. 185 (2005), no. 3, 267-285, DOI 10.4064/fm185-3-5. MR2161407 (2006c:37046)

[17] Robert L. Devaney, Cantor webs in the parameter and dynamical planes of rational maps, Holomorphic dynamics and renormalization, Fields Inst. Commun., vol. 53, Amer. Math. Soc., Providence, RI, 2008, pp. 105-123. MR2477420 (2009m:37130)

[18] Robert L. Devaney, Cantor sets of circles of Sierpiński curve Julia sets, Ergodic Theory Dynam. Systems 27 (2007), no. 5, 1525-1539, DOI 10.1017/S0143385707000156. MR2358976 (2008i:37087)

[19] Robert L. Devaney, Cantor necklaces and structurally unstable Sierpiński curve Julia sets for rational maps, Qual. Theory Dyn. Syst. 5 (2004), no. 2, 337-359, DOI 10.1007/BF02972685. MR2275444 (2007i:37091)

[20] Devaney, R. L., Cantor, Mandelbrot, Sierpiński Trees in the Parameter Planes for Rational Maps. To appear.

[21] Robert L. Devaney and Antonio Garijo, Julia sets converging to the unit disk, Proc. Amer. Math. Soc. 136 (2008), no. 3, 981-988 (electronic), DOI 10.1090/S0002-9939-07-09084-3. MR2361872(2008k:37098)

[22] Devaney, R. L. and Kozma, R. T. Julia sets converging to filled quadratic Julia sets. To appear in Ergod. Th. \& Dynan. Sys.

[23] Robert L. Devaney, Daniel M. Look, and David Uminsky, The escape trichotomy for singularly perturbed rational maps, Indiana Univ. Math. J. 54 (2005), no. 6, 1621-1634, DOI 10.1512/iumj.2005.54.2615. MR2189680(2006i:37105)

[24] Robert L. Devaney and Sebastian M. Marotta, The McMullen domain: rings around the boundary, Trans. Amer. Math. Soc. 359 (2007), no. 7, 3251-3273 (electronic), DOI 10.1090/S0002-9947-07-04137-2. MR2299454 (2008b:37078)

[25] Robert L. Devaney and Mark Morabito, Limiting Julia Sets for Singularly Perturbed Rational Maps, Internat. J. Bifur. Chaos Appl. Sci. Engrg. 18 (2008), 3175-3181. MR2482381 (2010c:37105) 
[26] Robert L. Devaney, Mónica Moreno Rocha, and Stefan Siegmund, Rational maps with generalized Sierpiński gasket Julia sets, Topology Appl. 154 (2007), no. 1, 11-27, DOI 10.1016/j.topol.2006.03.024. MR2271770 (2008h:37047)

[27] Robert L. Devaney and Kevin M. Pilgrim, Dynamic classification of escape time Sierpiński curve Julia sets, Fund. Math. 202 (2009), no. 2, 181-198, DOI 10.4064/fm202-2-5. MR:2506193 (2010f:37081)

[28] Devaney, R. L., Russell, E. D. Connectivity of Julia Sets for Singularly Perturbed Rational Maps. To appear.

[29] Adrien Douady, Systèmes dynamiques holomorphes, Bourbaki seminar, Vol. 1982/83, Astérisque, vol. 105, Soc. Math. France, Paris, 1983, pp. 39-63 (French). MR728980 (85h:58090)

[30] Adrien Douady and John H. Hubbard, A proof of Thurston's topological characterization of rational functions, Acta Math. 171 (1993), no. 2, 263-297, DOI 10.1007/BF02392534. MR $1251582(94 \mathrm{j}: 58143)$

[31] A. Douady and J. H. Hubbard, Étude dynamique des polynômes complexes. Partie I, Publications Mathématiques d'Orsay [Mathematical Publications of Orsay], vol. 84, Université de Paris-Sud, Département de Mathématiques, Orsay, 1984 (French). MR762431 (87f:58072a)

[32] Fatou, P. Sur les solutions uniformes de certaines equations fonctionelles. C. R. Acad. Sci. Paris 143 (1906), 546-548.

[33] Fitzgibbon, E. and Silvestri, S. Rational Maps: Julia Sets of Accessible Mandelbrot Sets Are Not Homeomorphic. To appear.

[34] Antonio Garijo, Xavier Jarque, and Mónica Moreno Rocha, Non-landing hairs in Sierpiński curve Julia sets of transcendental entire maps, Fund. Math. 214 (2011), no. 2, 135-160, DOI 10.4064/fm214-2-3. MR.2845719 (2012j:37072)

[35] Antonio Garijo and Sebastian M. Marotta, Singular perturbations of $z^{n}$ with a pole on the unit circle, J. Difference Equ. Appl. 16 (2010), no. 5-6, 573-595, DOI 10.1080/10236190903257842. MR2642467 (2011j:37079)

[36] Garijo, A., Marotta, S. and Russell, E. D. Singular Perturbations in the Quadratic Family with Multiple Poles. To appear in J. Diff. Eq. Appl.

[37] E. J. Hinch, Perturbation methods, Cambridge Texts in Applied Mathematics, Cambridge University Press, Cambridge, 1991. MR1138727 (93a:34002)

[38] Jane M. Hawkins and Daniel M. Look, Locally Sierpiński Julia sets of Weierstrass elliptic $\wp$ functions, Internat. J. Bifur. Chaos Appl. Sci. Engrg. 16 (2006), no. 5, 1505-1520, DOI 10.1142/S0218127406015453. MR2254870 (2007h:37068)

[39] Mark H. Holmes, Introduction to perturbation methods, Texts in Applied Mathematics, vol. 20, Springer-Verlag, New York, 1995. MR1351250 (96j:34095)

[40] Julia, G. Memoire sur l'itération des Fonctions Rationelles. J. Math. Pure Appl. 8 (1918), 47-245.

[41] Daniel M. Look, Sierpiński carpets as Julia sets for imaginary 3-circle inversions, J. Difference Equ. Appl. 16 (2010), no. 5-6, 705-713, DOI 10.1080/10236190903203911. MR2675601 (2011f:37082)

[42] R. Mañé, P. Sad, and D. Sullivan, On the dynamics of rational maps, Ann. Sci. École Norm. Sup. (4) 16 (1983), no. 2, 193-217. MR732343 (85j:58089)

[43] Sebastian M. Marotta, Singular perturbations in the quadratic family, J. Difference Equ. Appl. 14 (2008), no. 6, 581-595, DOI 10.1080/10236190701702429. MR2417010 (2010b:37128)

[44] Sebastian M. Marotta, Singular perturbations of $z^{n}$ with multiple poles, Internat. J. Bifur. Chaos Appl. Sci. Engrg. 18 (2008), no. 4, 1085-1100, DOI 10.1142/S0218127408020859. MR2422924(2010g:37067)

[45] Curt McMullen, Automorphisms of rational maps, Holomorphic functions and moduli, Vol. I (Berkeley, CA, 1986), Math. Sci. Res. Inst. Publ., vol. 10, Springer, New York, 1988, pp. 3160, DOI 10.1007/978-1-4613-9602-4_3. MR955807 (89m:58187)

[46] Curtis T. McMullen, Complex dynamics and renormalization, Annals of Mathematics Studies, vol. 135, Princeton University Press, Princeton, NJ, 1994. MR 1312365 (96b:58097)

[47] John Milnor, Dynamics in one complex variable, 3rd ed., Annals of Mathematics Studies, vol. 160, Princeton University Press, Princeton, NJ, 2006. MR2193309 (2006g:37070)

[48] John Milnor, Geometry and dynamics of quadratic rational maps. Experimental Math. 2 (1993), 37-83. 
[49] John Milnor, Geometry and dynamics of quadratic rational maps, Experiment. Math. 2 (1993), no. 1, 37-83. With an appendix by the author and Lei Tan. MR1246482 (96b:58094)

[50] Moreno Rocha, M. A Combinatorial Invariant for Escape Time Sierpiński Rational Maps. To appear.

[51] Kevin M. Pilgrim, Rational maps whose Fatou components are Jordan domains, Ergodic Theory Dynam. Systems 16 (1996), no. 6, 1323-1343, DOI 10.1017/S0143385700010051. MR:1424402 (97k:58141)

[52] Weiyuan Qiu, Xiaoguang Wang, and Yongcheng Yin, Dynamics of McMullen maps, Adv. Math. 229 (2012), no. 4, 2525-2577, DOI 10.1016/j.aim.2011.12.026. MR2880231

[53] Qiu, W., Roesch, P., Wang, X., and Yin, Y. Hyperbolic Components of McMullen Maps. To appear.

[54] P. Roesch, On capture zones for the family $f_{\lambda}(z)=z^{2}+\lambda / z^{2}$, Dynamics on the Riemann sphere, Eur. Math. Soc., Zürich, 2006, pp. 121-129, DOI 10.4171/011-1/6. MR2348958 (2008j:37101)

[55] Mitsuhiro Shishikura, On the quasiconformal surgery of rational functions, Ann. Sci. École Norm. Sup. (4) 20 (1987), no. 1, 1-29. MR892140 (88i:58099)

[56] Silvestri, S. Non Homeomorphic Julia Sets of Singularly Perturbed Rational Maps. To appear.

[57] Norbert Steinmetz, On the dynamics of the McMullen family $R(z)=z^{m}+\lambda / z^{l}$, Conform. Geom. Dyn. 10 (2006), 159-183 (electronic), DOI 10.1090/S1088-4173-06-00149-4. MR2261046(2008d:37079)

[58] Norbert Steinmetz, Sierpiński curve Julia sets of rational maps, Comput. Methods Funct. Theory 6 (2006), no. 2, 317-327. MR2291139(2007k:37059)

[59] Dennis Sullivan, Quasiconformal homeomorphisms and dynamics. I. Solution of the FatouJulia problem on wandering domains, Ann. of Math. (2) 122 (1985), no. 3, 401-418, DOI 10.2307/1971308. MR819553(87i:58103)

[60] Sutherland, S. Bad Polynomials for Newton's Method. In Linear and Complex Analysis, Springer-Verlag (1994).

[61] Lei Tan and Yongcheng Yin, Local connectivity of the Julia set for geometrically finite rational maps, Sci. China Ser. A 39 (1996), no. 1, 39-47. MR.1397233 (97g:58142)

[62] Thurston, W. Lecture Notes. CBMS Conference, University of Minnesota at Duluth, 1983.

[63] Ferdinand Verhulst, Methods and applications of singular perturbations, Texts in Applied Mathematics, vol. 50, Springer, New York, 2005. Boundary layers and multiple timescale dynamics. MR2148856 (2006k:34001)

[64] G. T. Whyburn, Topological characterization of the Sierpiński curve, Fund. Math. 45 (1958), 320-324. MR0099638 (20 \#6077)

[65] Jean-Christophe Yoccoz, Linéarisation des germes de difféomorphismes holomorphes de (C, 0), C. R. Acad. Sci. Paris Sér. I Math. 306 (1988), no. 1, 55-58 (French, with English summary). MR.929279(89i:58123)

Department of Mathematics, Boston University, Boston, Massachusetts 02215

E-mail address: bob@bu.edu 